\title{
A model of compact polymers on a family of three-dimensional fractal lattices
}

\author{
Dušanka Lekić ${ }^{1}$ and Sunčica Elezović-Hadžić ${ }^{2}$ \\ ${ }^{1}$ University of Banja Luka, Faculty of Science, Department of Physics, \\ M. Stojanovića 2, Banja Luka, Bosnia and Herzegovina \\ ${ }^{2}$ Faculty of Physics, University of Belgrade, P.O.Box 44, 11001 Belgrade, Serbia \\ E-mail: dusamar@netscape.net, suki@ff.bg.ac.rs
}

\begin{abstract}
We study Hamiltonian walks (HWs) on the family of three-dimensional modified Sierpinski gasket fractals, as a model for compact polymers in nonhomogeneous media in three dimensions. Each member of this fractal family is labeled with an integer $b \geq 2$. We apply an exact recursive method which allows for explicit enumeration of extremely long Hamiltonian walks of different types: closed and open, with end-points anywhere in the lattice, or with one or both ends fixed at the corner sites, as well as some Hamiltonian conformations consisting of two or three strands. Analyzing large sets of data obtained for $b=2,3$ and 4 , we find that numbers $Z_{N}$ of Hamiltonian walks, on fractal lattice with $N$ sites, for $N \gg 1$ behave as $Z_{N} \sim \omega^{N} \mu^{N^{\sigma}}$. The leading term $\omega^{N}$ is characterized by the value of the connectivity constant $\omega>1$, which depends on $b$, but not on the type of HW. In contrast to that, the stretched exponential term $\mu^{N^{\sigma}}$ depends on the type of HW through constant $\mu<1$, whereas exponent $\sigma$ is determined by $b$ alone. For larger $b$ values, using some general features of the applied recursive relations, without explicit enumeration of HWs, we argue that asymptotical behavior of $Z_{N}$ should be the same, with $\sigma=\ln 3 / \ln [b(b+1)(b+2) / 6]$, valid for all $b>2$. This differs from the formulae obtained recently for Hamiltonian walks on other fractal lattices, as well as from the formula expected for homogeneous lattices. We discuss the possible origin and implications of such result.
\end{abstract}

Keywords: solvable lattice models; polymers, copolymers, polyelectrolytes and biomolecular solutions; structures and conformations (theory)

\section{Introduction}

The fact that compact conformations of polymers are principal configurations of the native states of globular proteins makes them an important subset of all the physically accessible conformations. Since compact conformations occupy space as densely as possible, one of the simplest ways to model them is to use Hamiltonian walks (HWs) on a lattice, which are, by definition, self-avoiding walks (SAWs) that visit all the lattice sites exactly once [1]. In order to make this model more capable for capturing different features of various physical phenomena (such as protein melting [2] or protein folding [3]) local interactions can be introduced, but even in its simplest form, with no interactions 
taken into account, the problem of enumeration and classification of Hamiltonian walks proved to be extremely difficult.

Studies of Hamiltonian walks are primarily focused on finding the overall numbers $Z_{N}$ of open and closed HWs on lattice with $N$ sites. It is expected that limiting value of $\ln Z_{N} / N$ exists when $N \rightarrow \infty$, and its particular value

$$
\ln \omega=\lim _{N \rightarrow \infty} \frac{\ln Z_{N}}{N}
$$

corresponds to the configurational entropy per monomer (site). This means that to the lowest approximation, $Z_{N}$ behaves as $\omega^{N}$ and, therefore, the so-called connectivity constant $\omega>1$ can be interpreted as average number of steps available to the walker having already completed a large number of steps. The leading corrections to the exponential term are expected to have the power-law form $N^{a}$ (as in the case of ordinary self-avoiding walks), and the stretched exponential form $\mu^{N^{\sigma}}$, with $\mu<1$, so that $Z_{N}$ should scale as

$$
Z_{N} \sim \omega^{N} \mu^{N^{\sigma}} N^{a}
$$

Expectations of such forms are based on the exact studies of HWs on the Manhattan [4] and some fractal lattices [5-7], as well as on results obtained for closely related models of collapsed interacting self-avoiding walks on square [8-11] and cubic lattice [12]. In the case of collapsed SAWs appearance of the stretched exponential term, which is not present in the scaling form for non-interacting SAWs, was explained as a consequence of surface effects. Namely, a collapsed SAW forms a compact globule, with a sharp boundary separating it from the surrounding solvent, so that monomers on the boundary have smaller number of contacts with other monomers than those in the bulk of the globule, and therefore surface tension should arise. For homogeneous lattices one can assume that the boundary itself is homogeneous surface, and then straightforward arguments [13] lead to the conclusion that term $\mu^{N^{\sigma}}$, with $\sigma=(d-1) / d$ ( $d$ being the dimensionality of the lattice), should appear. Exact studies [9], series analysis of data obtained via exact enumeration [8,10], as well as Monte-Carlo simulations $[11,12]$ of collapsed SAWs on homogeneous lattices, certainly confirm existence of the stretched exponential term with the proposed formula for $\sigma$. Although it is believed that HW model corresponds to the interacting SAW model at temperature $T=0$, in spite of the continuous improvements of exact [14] and Monte Carlo [15] enumeration techniques, direct confirmation of scaling relation (1.1) for HWs on nonoriented homogeneous lattices has not been achieved yet. Apart from Manhattan lattice, scaling forms for Hamiltonian walks were obtained only for some fractal lattices, where stretched exponential corrections for both open and closed HWs were found only for $n$-simplex fractals with even $n$ [7]. Even in that cases, its presence cannot be explained using a simple generalization of the argument used for collapsed self-avoiding walks on homogeneous lattices. However, results of the studies on fractals suggest that stretched exponential term can be expected on lattices where larger number of entangled conformations is possible. In order to get a deeper insight into this issue, here we apply 
an exact recursive method for enumeration and classification of HWs on modified threedimensional Sierpinski gasket family of fractals. In contrast to most of the previously in this context studied fractals, which were embedded either in two-dimensional spaces or spaces with dimensionality higher than 3, each member of the family studied in the present paper is embedded in three-dimensional space. Therefore, HWs on these fractals can be understood as a toy model for compact polymers critical behaviour in realistic nonhomogeneous $3 \mathrm{~d}$ media, which, to the best of our knowledge, has not been studied so far.

The paper is organized as follows. Modified 3d Sierpinski gasket fractals, as well as the method itself, are described in the next section. Explicit forms of the recursion relations, obtained for the types of Hamiltonian walks which are needed for generation and enumeration of all closed conformations are presented for the first three members of the fractal family. Numerically analyzing these relations we find scaling form $\omega^{N} \mu_{C}^{N^{\sigma}}$. Using some general features of these relations (obtained in Appendix A and Appendix B], we argue that such form should be correct for the whole fractal family, and derive the closed-form formula for the exponent $\sigma$. In section 3 we extend the method to open HWs, explicitly apply it again on the first three fractals, and then generalize it. It turns out that the number of open HWs scales as $\omega^{N} \mu_{O}^{N^{\sigma}}$, with the same values of the connectivity constant $\omega$ and the exponent $\sigma$ as for the closed HWs, but with $\mu_{O} \neq \mu_{C}$. General features of the recursion relations for open HWs are derived in Appendix C and Appendix D. All the obtained results are summarized and discussed in the section 4, and some auxiliary considerations, needed for the discussion, are given in Appendix E.

\section{Closed Hamiltonian walks on modified three-dimensional Sierpinski gaskets}

Three-dimensional modified Sierpinski gasket (3d MSG) fractal is constructed recursively, starting with a unit tetrahedron. The first step of the construction, so called generator $G_{1}(b)$ of order $l=1$, is obtained by joining

$$
N_{G}=b(b+1)(b+2) / 6
$$

unit tetrahedrons into a $b$ times larger tetrahedral structure (see figure 1) in such a way that vertices of neighboring unit tetrahedrons are connected via infinitesimal junctions. Enlarging the generator $b$ times, and substituting the smallest tetrahedrons with $G_{1}(b)$, and then repeating this procedure $l$ times, one obtains $G_{l+1}(b)$ - generator of order $l+1$, which contains $N_{l+1}=4 N_{G}^{l+1}$ sites. The complete $3 \mathrm{~d}$ MSG fractal with parameter $b$ is obtained when $l \rightarrow \infty$, and its fractal dimension $d_{f}$ is equal to

$$
d_{f}=\ln [b(b+1)(b+2) / 6] / \ln b .
$$

One should note here that ordinary 3d Sierpinski gasket (SG) fractal with parameter $b$ is constructed in a similar way, with the only difference being that neighboring unit tetrahedrons in 3d SG lattice are not moved apart, as is the case for the 3d MSG fractal. 


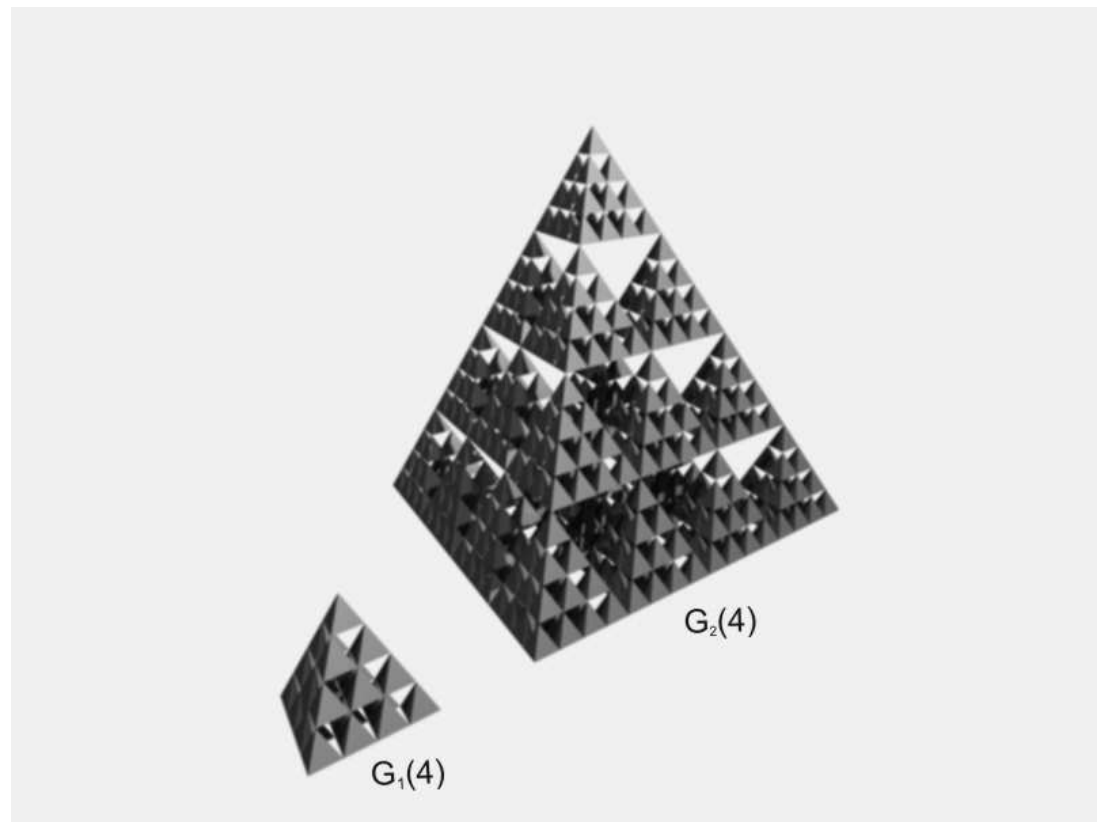

Figure 1. The first two steps of the construction of the 3d MSG fractal with $b=4$. Vertices of neighboring unit tetrahedrons are connected via infinitesimal junctions.

This small change does not alter the basic properties of the lattice, such as its fractal dimension, but it simplifies the complicated scheme needed for enumeration of closed Hamiltonian walks on 3d SG fractals [6] and allows for enumeration of extremely long open HWs on 3d MSG fractals with $b=2,3$, and 4. In addition, general conclusions about scaling forms of the numbers of HWs on $3 \mathrm{~d}$ MSG fractal with arbitrary $b>2$ can be derived, as will be explained in what follows.

By definition, each Hamiltonian walk on $G_{l}(b)$ structure visits all its $N_{l}$ sites exactly once. In figure 2 an example of closed Hamiltonian walk on the generator $G_{1}(3)$ is shown. One can notice that this HW can be decomposed into $N_{G}=10$ parts (within the ten unit tetrahedrons), which are either one- or two-stranded. This observation can easily be generalized to generators of higher order and any $b$ : any closed HW on $G_{l+1}(b)$ can be decomposed into $N_{G}$ parts within the same number of generators $G_{l}(b)$, from which the generator $G_{l+1}(b)$ is made of. These parts traverse each $G_{l}(b)$ either once or two times, and we shall call the corresponding configurations $B$ - or $E$-type HW steps, respectively, whereas numbers of HWs of these types within a $G_{l}(b)$ will be denoted by $B_{l}$ and $E_{l}$, respectively. Using these numbers, the overall number of closed HWs on $G_{l+1}(b)$ can be expressed as

$$
Z_{l+1}^{C}=\sum_{k=0}^{k_{C}} n_{k} B_{l}^{N_{G}-k} E_{l}^{k},
$$

where $n_{k}$ are the numbers of closed HWs configurations within $G_{l+1}(b)$, with $k$ steps of $E$-type, and $\left(N_{G}-k\right) B$-steps. For instance, the path presented in figure 2 is one of $Z_{1}^{C}$ possible closed HWs within the $G_{1}(3)$, contributing to the term $n_{2} B_{0}^{8} E_{0}^{2}$ in the 


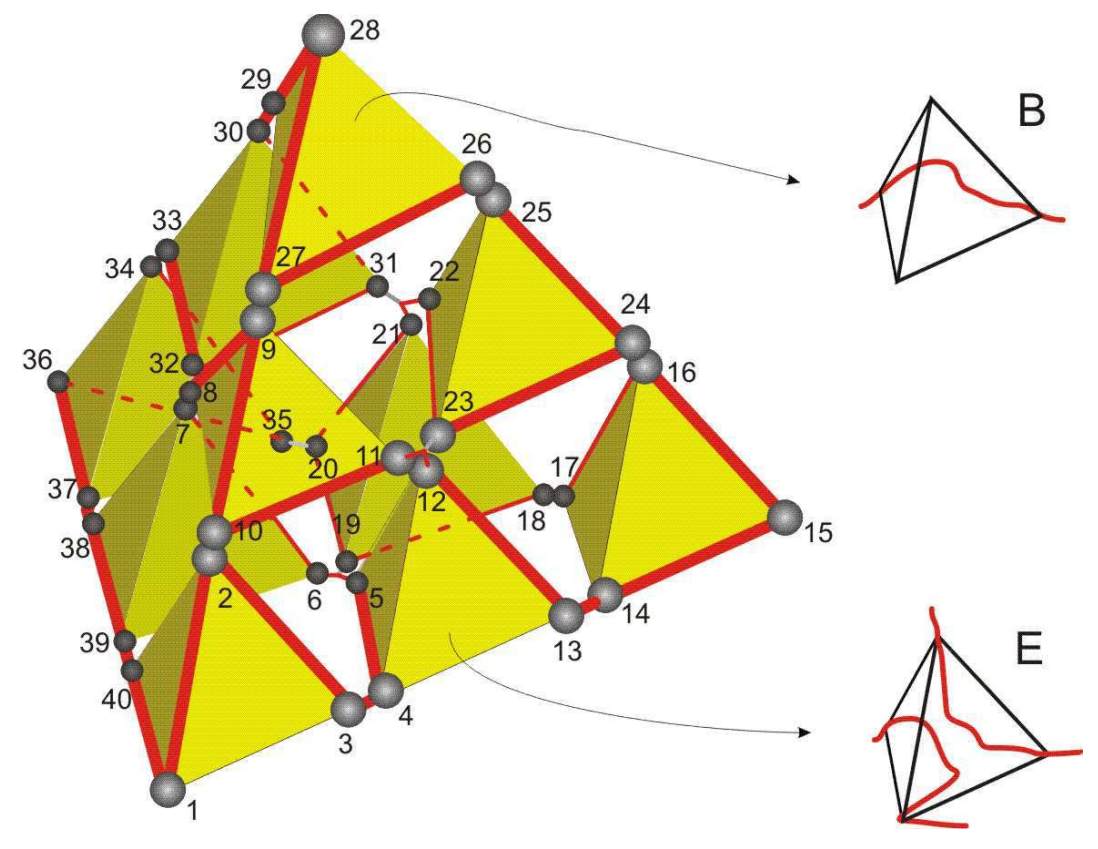

Figure 2. Example of closed Hamiltonian walk (red line) on the generator of the $3 \mathrm{~d} b=$ 3 MSG fractal. The generator consists of 40 vertices, which are numbered consecutively from the first (arbitrarily chosen) vertex visited by the HW, to the last one (which is connected with the first one). One can notice that HW conformation within the unit tetrahedron can be either one-stranded ( $B$-type), like the one connecting the sites 26 27-28-29, or two-stranded (E-type), like the conformation traversing the tetrahedron with vertices 4-5-12-13.

corresponding equation (2.3). The upper limit $k_{C}$ in the sum in (2.3) is equal to 0 for $b=2$, whereas for $b>2$ it can be shown ( see Appendix A) that

$$
k_{C}=\frac{1}{2}(b+1)(b+2)-8 .
$$

Due to the self-similarity of the lattices under study, the numbers $n_{k}$ do not depend on $l$, and, also, numbers $B_{l}$ and $E_{l}$ fulfil recursion relations of the following form:

$$
B_{l+1}=\sum_{k=k_{B}}^{N_{G}} m_{k} B_{l}^{k} E_{l}^{N_{G}-k}, \quad E_{l+1}=\sum_{k=k_{E}}^{N_{G}} p_{k} B_{l}^{k} E_{l}^{N_{G}-k},
$$

where coefficients $m_{k}$ and $p_{k}$ depend only on $k$ and $b$, and are positive integers for all $b>2$, including zero for $b=2$. For instance, for $b=2$ these relations ar $\ddagger$

$$
B_{l+1}=2 B_{l}^{4}+4 B_{l}^{3} E_{l}+6 B_{l}^{2} E_{l}^{2}, \quad E_{l+1}=B_{l}^{4}+4 B_{l}^{3} E_{l}+22 E_{l}^{4},
$$

and we were able to find the explicit form of relations (2.5) for $b=3$ and 4 also, by direct computer enumeration of possible HW conformations within the MSG generator. The corresponding coefficients $m_{k}$ and $p_{k}$ are presented in table 1, while for larger $b$ values they could not be reached within the reasonable time with the computer facilities $\ddagger$ Note that for $b=2$ recursion relations (2.5) are the same as for the 4-simplex fractal lattice [5]. 
Table 1. Coefficients appearing in recursion relations (2.5), found by direct computer enumeration of the corresponding HW conformations on 3d MSG fractals with $b=2,3,4$.

\begin{tabular}{ccccccccc}
\hline \multicolumn{3}{c}{$b=2$} & \multicolumn{3}{c}{$b=3$} & \multicolumn{3}{c}{$b=4$} \\
\hline$k$ & $m_{k}$ & $p_{k}$ & $k$ & $m_{k}$ & $p_{k}$ & $k$ & $m_{k}$ & $p_{k}$ \\
\hline 0 & - & 22 & 2 & - & 4308 & 7 & - & 26465392 \\
1 & - & 0 & 3 & - & 1936 & 8 & - & 99652120 \\
2 & 6 & 0 & 4 & - & 5808 & 9 & - & 151443088 \\
3 & 4 & 4 & 5 & 3192 & 1888 & 10 & 23848720 & 199987864 \\
4 & 2 & 1 & 6 & 848 & 2534 & 11 & 58605536 & 204194352 \\
- & - & - & 7 & 1728 & 1056 & 12 & 78351952 & 172479256 \\
- & - & - & 8 & 664 & 596 & 13 & 81469824 & 126633376 \\
- & - & - & 9 & 332 & 160 & 14 & 66418856 & 78454776 \\
- & - & - & 10 & 64 & 32 & 15 & 43526336 & 41200784 \\
- & - & - & - & - & - & 16 & 22989024 & 18548660 \\
- & - & - & - & - & - & 17 & 9642816 & 6662824 \\
- & - & - & - & - & - & 18 & 3032724 & 1901008 \\
- & - & - & - & - & - & 19 & 626056 & 397392 \\
- & - & - & - & - & - & 20 & 62434 & 42514 \\
\hline
\end{tabular}

available to us. However, one can show (see Appendix A) that for general $b>2$ lower limits of the sums in relations (2.5) are equal to

$$
k_{B}=N_{G}-k_{C}-3=\frac{1}{6}(b+1)(b+2)(b-3)+5, \quad k_{E}=k_{B}-3,
$$

which is a result essential for establishing some general conclusions, as will be explained in the following paragraphes.

Once the explicit form of recursion relations (2.3) and (2.5) is established, starting with the initial values $B_{0}=2$, and $E_{0}=1$, corresponding to the unit tetrahedron, one can calculate the numbers $B_{l}, E_{l}$, and subsequently the overall number $Z_{l+1}^{C}$ of closed HWs, in principle for any $l$. However, since these numbers quickly become extremely large, it is useful to introduce new variable $x_{l}=B_{l} / E_{l}$. Then, from (2.5) it follows that recursion relation for the variable $x_{l}$, for $b>2 \xi$, has the form

$$
x_{l+1}=x_{l}^{k_{B}-k_{E}} g\left(x_{l}\right)=x_{l}^{3} g\left(x_{l}\right), \quad g(x)=\frac{\sum_{k=0}^{k_{C}+3} m_{k_{B}+k} x^{k}}{\sum_{k=0}^{k_{C}+6} p_{k_{E}+k} x^{k}} .
$$

Numerical analysis of this recursion relation for $b=3$ and $b=4$ cases, shows that starting with the initial value $x_{0}=2, x_{l}$ quickly tends to 0 , as $l$ grows. Assuming that this is correct for general $b>2$, for large values of $l$ the above equation can be approximated as

$$
x_{l+1} \approx \frac{m_{k_{B}}}{p_{k_{E}}} x_{l}^{3}
$$

$\S$ The corresponding analysis of the $b=2$ case is the same as for 4 -simplex fractal lattice, which is given in [5]. 
Table 2. Values of relevant constants appearing in the scaling forms (2.17) and (3.13) of the overall numbers of HWs on $3 \mathrm{~d}$ MSG fractals, with $b=2,3,4$, together with the corresponding values of the fractal dimension $d_{f}$.

\begin{tabular}{rrrllllll}
\hline$b$ & $d_{f}$ & $k_{C}$ & $\lambda$ & $\omega$ & $\mu_{C}$ & $\mu_{O}$ & $\sigma$ & $1 / d_{f}$ \\
\hline 2 & $\ln 4 / \ln 2$ & 0 & 0.8366 & 1.0876 & 0.8366 & 0.9147 & 0.5 & 0.5 \\
3 & $\ln 10 / \ln 3$ & 2 & 0.8835 & 1.4404 & 0.8963 & 0.9554 & 0.4471 & 0.4471 \\
4 & $\ln 20 / \ln 4$ & 7 & 0.8639 & 1.4686 & 0.8696 & 0.9496 & 0.3667 & 0.4628 \\
\hline
\end{tabular}

from which follows

$$
x_{l} \sim \lambda^{3^{l}}
$$

where $\lambda$ is some constant, whose value depends on $b$, but it is always less than 1 . For $b=2$ the corresponding relation is $x_{l} \sim \lambda^{2^{l}}[5]$, and particular values of $\lambda$ for $b=2,3$ and 4 cases are given in table 2 .

Recursion relation for numbers $E_{l}$ of two-stranded HWs (2.5), with the variable $x_{l}=B_{l} / E_{l}$ obtains the form

$$
E_{l+1}=E_{l}^{N_{G}} x_{l}^{k_{E}} f\left(x_{l}\right), \quad f(x)=\sum_{k=0}^{k_{C}+6} p_{k+k_{E}} x^{k},
$$

from which one gets

$$
\frac{\ln E_{l+1}}{4 N_{G}^{l+1}}=\frac{\ln E_{l}}{4 N_{G}^{l}}+k_{E} \frac{\ln x_{l}}{4 N_{G}^{l+1}}+\frac{\ln f\left(x_{l}\right)}{4 N_{G}^{l+1}} .
$$

Numerically iterating this recursion relation, together with (2.8), one finds that

$$
\lim _{l \rightarrow \infty} \frac{\ln E_{l}}{4 N_{G}^{l}}=\ln \omega
$$

where $\omega$ (see table 2) is constant larger than 1. On the other hand, the overall number $Z_{l+1}^{C}(2.3)$ of closed HWs on the generator of order $l+1$ can be expressed as

$$
Z_{l+1}^{C}=E_{l}^{N_{G}} x_{l}^{N_{G}-k_{C}} h\left(x_{l}\right), \quad h(x)=\sum_{k=0}^{k_{C}} n_{k_{C}-k} x^{k},
$$

so that

$$
\frac{\ln Z_{l+1}^{C}}{4 N_{G}^{l+1}}=\frac{\ln E_{l}}{4 N_{G}^{l}}+\frac{N_{G}-k_{C}}{4 N_{G}} \frac{\ln x_{l}}{N_{G}^{l}}+\frac{\ln h\left(x_{l}\right)}{4 N_{G}^{l+1}} .
$$

From the asymptotical behavior (2.10) of the number $x_{l}$, and from the fact that $h(x)$ tends to constant value $n_{k_{C}}>0$ when $x \rightarrow 0$, it then follows that

$$
\lim _{l \rightarrow \infty} \frac{\ln Z_{l}^{C}}{N_{l}}=\lim _{l \rightarrow \infty} \frac{\ln E_{l}}{4 N_{G}^{l}}=\ln \omega,
$$

where $N_{l}=4 N_{G}^{l}$ is overall number of vertices within the generator of order $l$.

To find the leading-order correction to the asymptotic behavior of $Z_{l}^{C}$ we first introduce variable

$$
y_{l}=\frac{\ln E_{l}}{4 N_{G}^{l}}=\frac{\ln E_{l}}{N_{l}},
$$


which, as follows from (2.12), satisfies the relation

$$
y_{l}=\sum_{k=0}^{l-1}\left(y_{k+1}-y_{k}\right)+y_{0}=\sum_{k=0}^{l-1} \frac{1}{N_{k+1}}\left[k_{E} \ln x_{k}+\ln f\left(x_{k}\right)\right] .
$$

Then, using (2.13), one obtains

$$
y_{l}=\ln \omega-\sum_{k=l}^{\infty} \frac{1}{N_{k+1}}\left[k_{E} \ln x_{k}+\ln f\left(x_{k}\right)\right],
$$

from which, taking into account the large $k$ behavior (2.10) of $x_{k}$, it follows that

$$
y_{l} \approx \ln \omega-\frac{1}{N_{l}} \frac{k_{E}}{N_{G}-3} 3^{l} \ln \lambda-\frac{\text { const }}{N_{l}}, \quad l \gg 1 .
$$

Substituting this relation into (2.15) one derives

$$
\ln Z_{l}^{C} \approx N_{l} \ln \omega+N_{l}^{\sigma} \ln \mu_{C}, \quad \text { i.e. } \quad Z_{l}^{C} \sim \omega^{N_{l}} \mu_{C}^{N_{l}^{\sigma}}
$$

with

$$
\sigma=\frac{\ln 3}{\ln N_{G}}=\frac{\ln 3}{\ln [b(b+1)(b+2) / 6]},
$$

and

$$
\mu_{C}=\lambda^{A}, \quad A=\frac{N_{G}+k_{C}}{N_{G}-3} 4^{-\sigma} .
$$

At the end of this section we want to emphasize that the crucial step in preceding derivation, which led to the scaling form (2.17), with the value of exponent $\sigma$ given by formula (2.18), was the assumption that $x_{l}$ tends to 0 for general $b$ (explicitly confirmed only up to $b=4$ ). Its direct consequence is approximate difference equation (2.9), which is obtained from the exact relation (2.8). On the other hand, the key ingredient of that relation is the fact that $k_{B}-k_{E}=3$, due to which $x_{l}$ behaves as $\lambda^{3^{l}}$, for $l \gg 1$. In that sense, exact expressions for $k_{B}$ and $k_{E}$ (2.7), obtained in Appendix A for general $b>2$, are essential for establishing the scaling form (2.17), whereas the particular values of the coefficients $m_{k}$ and $p_{k}$ of the recursion relations (2.5) do not affect neither its general form, nor the value of $\sigma$. However, whether $x_{l}$ tends to 0 or not certainly depends on the values of $m_{k}$ and $p_{k}$. Analyzing data given in table 1 for $b=3$ and $b=4$, one can observe that $p_{k_{E}+k}>m_{k_{E}+k+1}$, for $2 \leq k \leq k_{C}+5$. It can be shown (see Appendix B that such inequality for general $b>2$ is sufficient to prove that numbers $x_{l}$, which satisfy difference equation (2.8) with the initial condition $x_{0}=2$, tend to 0 when $l \rightarrow \infty$. Unfortunately, the inequality itself we were not able to prove, but, since it seems plausible, we think that scaling relation (2.17) can be accepted as valid for general $b$.

\section{Open Hamiltonian walks on modified three-dimensional Sierpinski gaskets}

Any open HW on generator $G_{l+1}(b)$ of order $l+1$ can be decomposed into $N_{G}$ parts within its $N_{G}$ constitutive generators $G_{l}(b)$ of order $l$. The parts which contain ending points (see figure 3) can be of four different types: 
- A-type, which consists of one HW, with one end at one vertex of $G_{l}(b)$ and the other end at any other site of $G_{l}(b)$, including its vertices;

- $C$-type, composed of two non-intersecting SAWs, one with both ends at the vertices of the $G_{l}(b)$, and the other with one end at the third vertex of the $G_{l}(b)$ and the second anywhere within it - these two SAWs together visit all the sites of the $G_{l}(b)$;

- D-type, consisting of two non-intersecting SAWs, each of them starting at different vertex of $G_{l}(b)$ and ending anywhere within it, in such a way that all sites of $G_{l}(b)$ are visited;

- H-type, comprised of three non-intersecting SAWs, each of them starting at different vertex of $G_{l}(b)$, one ending at the fourth vertex, and the remaining two anywhere within $G_{l}(b)$ - again, all sites of $G_{l}(b)$ should be visited by these three SAWs.

$A$ - and $C$-type configurations have one dangling end, therefore we shall call them oneleg configurations (steps), whereas $D$ - and $H$-configurations, with two dangling ends, will be called two-leg steps. If both end-points of the complete HW lie in the same $G_{l}(b)$, that $G_{l}(b)$ contains a two-leg step (of $D$ - or $H$-type), whereas the remaining $N_{G}-1$ generators $G_{l}(b)$ contain either $B$ - or $E$-step. Otherwise, when end-points are in two different $G_{l}(b)$ generators, the complete open HW has two one-leg steps (of $A$ or $C$-type), and the remaining $N_{G}-2$ parts of the walk are of either $B$ - or $E$-type. Therefore, one concludes that overall number $Z_{l+1}^{O}$ of open HWs within $G_{l+1}(b)$ is equal to

$$
Z_{l+1}^{O}=A_{l}^{2} F_{A A}+A_{l} C_{l} F_{A C}+C_{l}^{2} F_{C C}+D_{l} S_{D}+H_{l} S_{H},
$$

where $F_{A A}, F_{A C}, F_{C C}, S_{D}$ and $S_{H}$ are polynomials in $B_{l}$ and $E_{l}$, of power $N_{G}-2$ or $N_{G}-1$, and $A_{l}, C_{l}, D_{l}$ and $H_{l}$ are overall numbers of corresponding type HWs within $G_{l}(b)$ generator. Numbers of one-leg conformations fulfill recursion relations of the following form:

$$
\begin{aligned}
& A_{l+1}=R_{11}\left(B_{l}, E_{l}\right) A_{l}+R_{12}\left(B_{l}, E_{l}\right) C_{l}, \\
& C_{l+1}=R_{21}\left(B_{l}, E_{l}\right) A_{l}+R_{22}\left(B_{l}, E_{l}\right) C_{l},
\end{aligned}
$$

with

$$
R_{i j}(B, E)=\sum_{k=0}^{k_{i j}} r_{k}^{i j} B^{N_{G}-1-k} E^{k},
$$

while numbers of two-leg conformations obey relations of the form

$$
\begin{aligned}
& D_{l+1}=d_{D} D_{l}+d_{H} H_{l}+d_{A A} A_{l}^{2}+d_{A C} A_{l} C_{l}+d_{C C} C_{l}^{2}, \\
& H_{l+1}=h_{D} D_{l}+h_{H} H_{l}+h_{A A} A_{l}^{2}+h_{A C} A_{l} C_{l}+h_{C C} C_{l}^{2},
\end{aligned}
$$

with $d$ and $h$ being some polynomials in $B_{l}$ and $E_{l}$. For instance, for $b=2$ relations (3.1) and (3.2) are:

$$
\begin{aligned}
& Z_{l+1}^{O}=12 B_{l}^{2}\left(A_{l}^{2}+2 A_{l} C_{l}+3 C_{l}^{2}+B_{l} D_{l}\right), \\
& A_{l+1}=\left(6 B_{l}^{3}+6 B_{l}^{2} E_{l}\right) A_{l}+\left(12 B_{l}^{3}+18 B_{l}^{2} E_{l}\right) C_{l}, \\
& C_{l+1}=\left(B_{l}^{3}+3 B_{l}^{2} E_{l}\right) A_{l}+\left(3 B_{l}^{3}+12 B_{l}^{2} E_{l}+16 B_{l} E_{l}^{2}+16 E_{l}^{3}\right) C_{l} .
\end{aligned}
$$




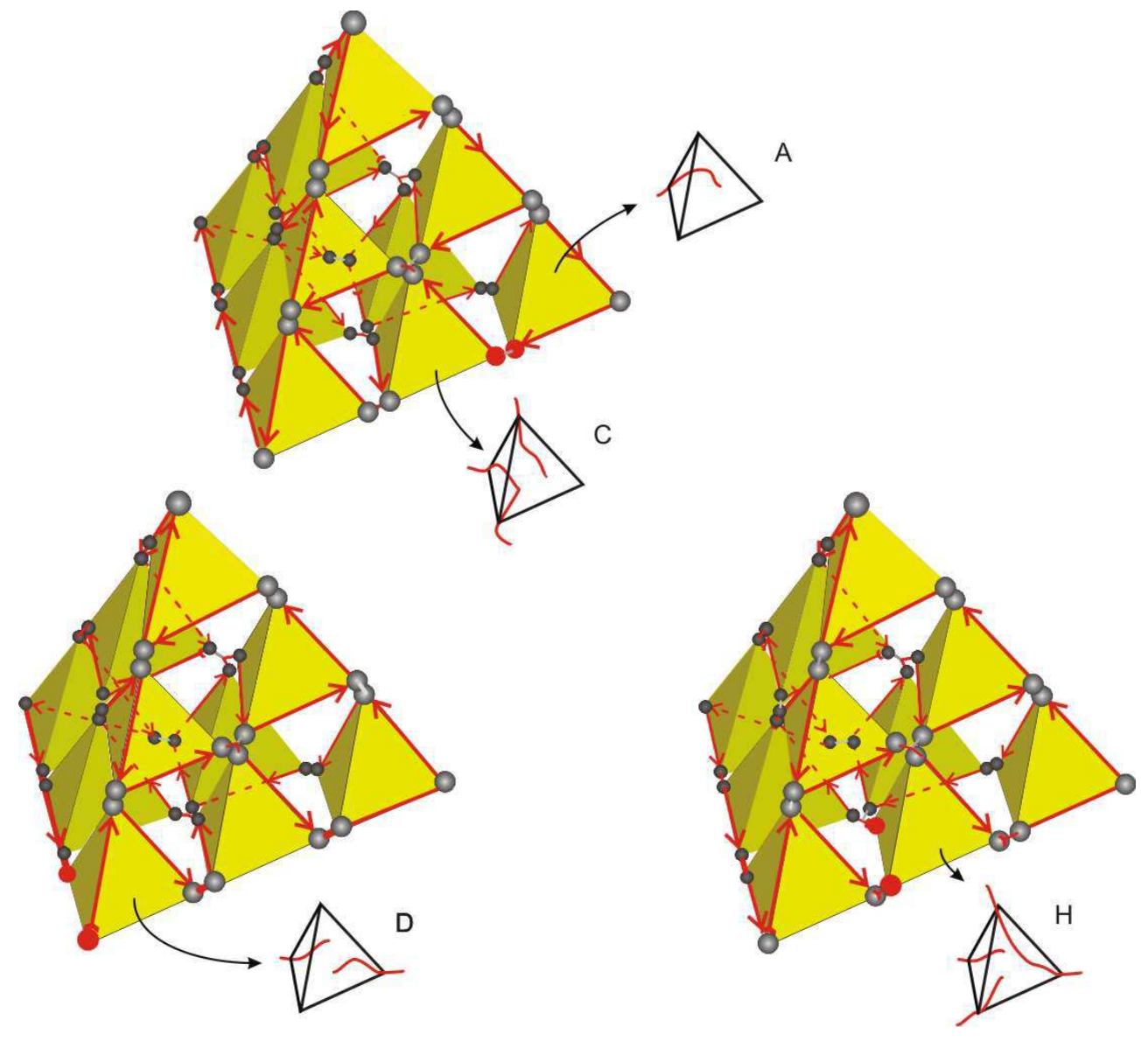

Figure 3. Three examples of open HW (oriented red lines, where the only meaning of arrows is to serve as guides to the eye) on the generator of the $3 \mathrm{~d} b=3 \mathrm{MSG}$ fractal. Points at which HW begins or terminates are marked as full red circles, for the sake of better recognition. If both ends of the walk are in the same tetrahedron the twoleg HW conformation within that tetrahedron is either of $D$ - or $H$-type. Otherwise, if end-points of the walk belong to different tetrahedrons, the corresponding one-leg conformations are of $A$ - or $C$-type.

They coincide with the corresponding relations for 4-simplex fractal lattice, as well as does the complete further analysis, which can be seen in [7]. In the remaining part of this section we present the general analysis for $b>23 \mathrm{~d}$ MSG fractals.

For general $b>2$ it can be shown (see Appendix C) that polynomials $F$ and $S$, appearing in (3.1), have the form

$$
\begin{aligned}
& F_{X Y}(B, E)=\sum_{k=0}^{k_{C}+1} z_{k}^{X Y} B^{N_{G}-k-2} E^{k}, \quad X Y=A A, A C, C C \\
& S_{D}(B, E)=\sum_{k=0}^{k_{C}} z_{k}^{D} B^{N_{G}-k-1} E^{k}, \quad S_{H}(B, E)=\sum_{k=0}^{k_{C}-1} z_{k}^{H} B^{N_{G}-k-1} E^{k}
\end{aligned}
$$

Starting with the initial values for the numbers $B_{l}, E_{l}, A_{l}, C_{l}, D_{l}$, and $H_{l}$, and using the recursive relations (2.5), (3.2), and (3.4), one can calculate these numbers in principle for any $l$, and substituting them into (3.1), eventually find the overall number $Z_{l+1}^{O}$ of 
open HWs. Since all these numbers increase rapidly with $l$, it is useful to introduce variables

$$
u_{l}=\frac{A_{l}}{E_{l}}, \quad v_{l}=\frac{C_{l}}{E_{l}}, \quad w_{l}=\frac{D_{l}}{E_{l}}, \quad q_{l}=\frac{H_{l}}{E_{l}},
$$

in addition to already defined $x_{l}=B_{l} / E_{l}$. With these variables $Z_{l+1}^{O}$ can be rewritten as

$$
\begin{gathered}
Z_{l+1}^{O}=E_{l}^{N_{G}} x_{l}^{N_{G}-k_{C}-3}\left[\sum_{k=0}^{k_{C}+1}\left(z_{k_{C}+1-k}^{A A} u_{l}^{2}+z_{k_{C}+1-k}^{A C} u_{l} v_{l}+z_{k_{C}+1-k}^{C C} v_{l}^{2}\right) x_{l}^{k}\right. \\
\left.+w_{l} x_{l}^{2} \sum_{k=0}^{k_{C}} z_{k_{C}-k}^{D} x_{l}^{k}+q_{l} x_{l}^{3} \sum_{k=0}^{k_{C}-1} z_{k_{C}-k-1}^{H} x_{l}^{k}\right]
\end{gathered}
$$

and explicitly calculated using corresponding recursion relations for $u_{l}, v_{l}, w_{l}$, and $q_{l}$, simultaneously with (2.8) and (2.11). In Appendix C it is shown that the upper limits of the sums in (3.3) are equal to

$$
k_{11}=k_{12}=k_{C}+2, \quad k_{21}=k_{22}=k_{C}+5,
$$

so that by dividing (3.2) with recursion relation for $E_{l}$, given in (2.5), for $u_{l}$ and $v_{l}$ one obtains recursion relations

$$
\left(\begin{array}{l}
u_{l+1} \\
v_{l+1}
\end{array}\right)=\left(\begin{array}{ll}
m_{11} & m_{12} \\
m_{21} & m_{22}
\end{array}\right)\left(\begin{array}{l}
u_{l} \\
v_{l}
\end{array}\right)
$$

where $m_{i j}$ are functions of $x_{l}$ of the following form:

$m_{1 i}(x)=\frac{x^{3}}{f(x)} \sum_{k=0}^{k_{C}+2} r_{k_{C}+2-k}^{1 i} x^{k}, \quad m_{2 i}(x)=\frac{1}{f(x)} \sum_{k=0}^{k_{C}+5} r_{k_{C}+5-k}^{2 i} x^{k}, \quad i=1,2$,

and $f(x)$ is defined in (2.11). Since $x_{l} \rightarrow 0$ and $f\left(x_{l}\right) \rightarrow$ const $\neq 0$ when $l \rightarrow \infty$, it follows that $m_{1 i}\left(x_{l}\right) \rightarrow 0, m_{2 i}\left(x_{l}\right) \rightarrow$ const $\neq 0$, implying that $u_{l}$ tends to 0 , and $v_{l}$ to some constant value. This is indeed correct for $b=3$ and $b=4$ cases, for which we managed to find the complete set of coefficient $r_{k}^{i j}$ (see table 3 ). Starting with the initial values $A_{0}=6, C_{0}=2$, i.e. $u_{0}=6$ and $v_{0}=2$, and numerically iterating relations (3.9), for both $b=3$ and $b=4$, already after five iterations one obtains limiting values of $v_{l}$ : $441.32 \ldots$ for $b=3$, and $3538.91 \ldots$ for $b=4$.

In Appendix D it is shown that recursion relations for the variables $w_{l}$ and $q_{l}$ can be put into the following matrix form

$$
\left(\begin{array}{c}
w_{l+1} \\
q_{l+1}
\end{array}\right)=\left(\begin{array}{ll}
p_{11} & p_{12} \\
p_{21} & p_{22}
\end{array}\right)\left(\begin{array}{c}
w_{l} \\
q_{l}
\end{array}\right)+\left(\begin{array}{c}
t_{11} u_{l}^{2}+t_{12} u_{l} v_{l}+t_{13} v_{l}^{2} \\
t_{21} u_{l}^{2}+t_{22} u_{l} v_{l}+t_{23} v_{l}^{2}
\end{array}\right),
$$

where $p_{i j}$ and $t_{i j}$ are functions of $x_{l}$ of the form

$$
\begin{aligned}
& p_{11}(x)=\frac{x^{2}}{f(x)} \sum_{k=0}^{k_{C}+3}\left(k+k_{B}\right) m_{k+k_{B}} x^{k}, \quad p_{12}(x)=\frac{2 x^{3}}{f(x)} \sum_{k=0}^{k_{C}+2}\left(k_{C}+3-k\right) m_{k+k_{B}} x^{k}, \\
& p_{21}(x)=\frac{1}{2 x f(x)} \sum_{k=0}^{k_{C}+6}\left(k+k_{E}\right) p_{k+k_{E}} x^{k}, \quad p_{22}(x)=\frac{1}{f(x)} \sum_{k=0}^{k_{C}+6}\left(k_{C}+6-k\right) p_{k+k_{E}} x^{k},
\end{aligned}
$$


Table 3. Coefficients of the polynomials $R_{i j}$ (3.3), appearing in recursion relations (3.2), found by direct computer enumeration of the corresponding HW conformations on 3d MSG fractals with $b=3$ and 4 .

\begin{tabular}{|c|c|c|c|c|c|c|c|c|}
\hline \multirow[b]{2}{*}{$k$} & \multicolumn{4}{|c|}{$b=3$} & \multicolumn{4}{|c|}{$b=4$} \\
\hline & $r_{k}^{11}$ & $r_{k}^{12}$ & $r_{k}^{21}$ & $r_{k}^{22}$ & $r_{k}^{11}$ & $r_{k}^{12}$ & $r_{k}^{21}$ & $r_{k}^{22}$ \\
\hline 0 & 378 & 1050 & 202 & 620 & 726000 & 2078460 & 454340 & 1343670 \\
\hline 1 & 1722 & 5256 & 798 & 2854 & 6785652 & 20162052 & 4124238 & 12825924 \\
\hline 2 & 2748 & 8964 & 1962 & 8696 & 30159948 & 92803776 & 18522682 & 61201186 \\
\hline 3 & 3588 & 15144 & 2912 & 15172 & 85107696 & 270289488 & 56914854 & 200289608 \\
\hline 4 & 2976 & 9576 & 3126 & 24064 & 171919920 & 559983864 & 135608360 & 503818972 \\
\hline 5 & - & - & 4632 & 23684 & 265804824 & 875876784 & 260879696 & 1011455276 \\
\hline 6 & - & - & 2420 & 27088 & 319608912 & 1041132024 & 412200376 & 1653387800 \\
\hline 7 & - & - & 1304 & 4308 & 290292720 & 895898352 & 548029844 & 2243585880 \\
\hline 8 & - & - & - & - & 185693208 & 493595664 & 612939852 & 2479455304 \\
\hline 9 & - & - & - & - & 51662736 & 71546160 & 552106184 & 1451395108 \\
\hline 10 & - & - & - & - & - & - & 407928692 & 1451395108 \\
\hline 11 & - & - & - & - & - & - & 226652724 & 546796688 \\
\hline 12 & - & - & - & - & - & - & 68954256 & 26465392 \\
\hline
\end{tabular}

$t_{1 i}(x)=\frac{1}{f(x)} \sum_{k=0}^{k_{C}+4} s_{k_{C}+4-k}^{X Y} x^{k}, \quad t_{21}(x)=\frac{1}{x^{3} f(x)} \sum_{k=0}^{k_{C}+7} o_{k_{C}+7-k}^{A A} x^{k}$,

$t_{22}(x)=\frac{1}{x^{2} f(x)} \sum_{k=0}^{k_{C}+6} o_{k_{C}+6-k}^{A C} x^{k}, \quad t_{23}(x)=\frac{1}{x f(x)} \sum_{k=0}^{k_{C}+5} o_{k_{C}+5-k}^{C C} x^{k}$,

with $s_{k}^{X Y}$ and $o_{k}^{X Y}$ being some positive constant integers. Using these relations, one can calculate $w_{l}$ and $q_{l}$, and by putting them, together with $x_{l}, u_{l}, v_{l}$ and $E_{l}$, into (3.7), finally can evaluate $Z_{l}^{O}$, for any $l$.

In order to find asymptotic behavior of $Z_{l}^{O}$, we first notice that relations (3.10) can be combined with the relation (2.8) for $x_{l}$, so that one can establish recursion relations for $w_{l} x_{l}^{2}$ and $q_{l} x_{l}^{3}$, terms through which variables $w_{l}$ and $q_{l}$ appear in (3.7). Using the facts that $x_{l} \rightarrow 0, f\left(x_{l}\right) \rightarrow p_{k_{E}}, u_{l} \rightarrow 0$, and $v_{l} \rightarrow$ const, when $l \rightarrow \infty$, it can be shown that

$$
\left(\begin{array}{c}
w_{l+1} x_{l+1}^{2} \\
q_{l+1} x_{l+1}^{3}
\end{array}\right) \approx x_{l}^{6}\left[\left(\begin{array}{ll}
a_{11} & a_{12} \\
a_{21} & a_{22}
\end{array}\right)\left(\begin{array}{c}
w_{l} x_{l}^{2} \\
q_{l} x_{l}^{3}
\end{array}\right)+\left(\begin{array}{c}
b_{13} v_{l}^{2} \\
0
\end{array}\right)\right],
$$

where $a_{i j}$ and $b_{13}$ are some constants (see Appendix D). This relation implies that $w_{l} x_{l}^{2}$ and $q_{l} x_{l}^{3}$ tend to zero as $l \rightarrow \infty$, and consequently, comparing the terms containing $w_{l} x_{l}^{2}$ and $q_{l} x_{l}^{3}$ with those with $u_{l}$ and $v_{l}$ in (3.7), one can conclude that

$Z_{l+1}^{O} \approx E_{l}^{N_{G}} x_{l}^{N_{G}-k_{C}-3} \sum_{k=0}^{k_{C}+1}\left(z_{k_{C}+1-k}^{A A} u_{l}^{2}+z_{k_{C}+1-k}^{A C} u_{l} v_{l}+z_{k_{C}+1-k}^{C C} v_{l}^{2}\right) x_{l}^{k}$.

This means that for $l \gg 1$ number $Z_{l+1}^{O}$ behaves as

$$
Z_{l+1}^{O} \sim E_{l}^{N_{G}} x_{l}^{N_{G}-k_{C}-3}
$$


so that, using (2.14), one obtains

$$
\frac{Z_{l+1}^{O}}{Z_{l+1}^{C}} \sim x_{l}^{-3} \sim \lambda^{-3^{l+1}} .
$$

Consequently, taking into account (2.17), it follows that overall number $Z_{l}^{O}$ of open HWs scales as

$$
Z_{l}^{O} \sim \omega^{N_{l}} \mu_{O}^{N_{l}^{\sigma}}, \quad \mu_{O}=\lambda^{B}, \quad B=\frac{k_{C}+3}{N_{G}-3} 4^{-\sigma},
$$

where, as in (2.18),$\sigma=\ln 3 / \ln N_{G}$, and particular values of $\omega, \mu_{O}$ and $\lambda$ are given in table 2 .

\section{Summary and Discussion}

In this paper we have analyzed asymptotic behavior of the numbers of open and closed Hamiltonian walks on three-dimensional modified Sierpinski gasket family of fractals. Numbers of extremely long HWs on these lattices can be generated by applying an exact recursive enumeration scheme, based on the fact that any HW on the $(l+1)$ th step of the construction of the 3d MSG fractal, $G_{l+1}(b)$, consists of $N_{G}=b(b+1)(b+2) / 6$ HWs within its $N_{G}$ constitutive $G_{l}(b)$ structures, which can be of one of six possible types: $A, B, C, D, E$, and $H$ (figure 2 and figure 3). Numbers of these HWs, $A_{l}, B_{l}$, $C_{l}, D_{l}, E_{l}$, and $H_{l}$, fulfil closed set of recursive relations, (2.5), (3.2) and (3.4), which, due to the self-similarity of the lattices under study, do not depend on $l$, and therefore can be obtained by explicit enumeration and classification of $\mathrm{HW}$ conformations on the first step of the fractal construction, $G_{1}(b)$. As $b$ grows, number of HW conformations rapidly increases already on $G_{1}(b)$, so that we have managed to find explicit form of the recursive relations only up to $b=4$. However, we have shown that for any $b>2$ these recursive relations have some features that enable general analysis, leading to conclusion that overall numbers $Z_{l}^{C}$ and $Z_{l}^{O}$ of closed and open HWs, respectively, on any 3d MSG fractal, scale with the number $N_{l}=4 N_{G}^{l}$ of the lattice sites as

$$
Z_{l}^{C} \sim \omega^{N_{l}}\left[\lambda^{\frac{N_{G}+k_{C}}{N_{G}-3} 4^{-\sigma}}\right]^{N_{l}^{\sigma}}, \quad Z_{l}^{O} \sim \omega^{N_{l}}\left[\lambda^{\frac{k_{C}+3}{N_{G}-3}} 4^{-\sigma}\right]^{N_{l}^{\sigma}},
$$

with $\sigma$ given by formula (2.18):

$$
\sigma=\frac{\ln 3}{\ln \frac{1}{6} b(b+1)(b+2)}=\frac{\ln 3}{\ln b} \frac{1}{d_{f}} .
$$

Constants $\omega$ and $\lambda$ can be obtained numerically, using relations

$$
\ln \omega=\lim _{l \rightarrow \infty} \frac{\ln E_{l}}{N_{l}}, \quad \ln \lambda=\lim _{l \rightarrow \infty} \frac{\ln \left(B_{l} / E_{l}\right)}{3^{l}},
$$

and their particular values for $b=2,3$, and 4 are given in table 2. Number $k_{C}=(b+1)(b+2) / 2-8$ represents the maximal number of $G_{l}(b)$ generators within the $G_{l+1}(b)$ they belong to, which are traversed by two-stranded $E$-type HW conformation (E-step) within any closed $H W$ on $G_{l+1}(b)$ (see equation (2.3) and Appendix A). As one can see, HW conformations that traverse $G_{l}(b)$, and recursion relations (2.5) for 
the corresponding numbers $B_{l}$ and $E_{l}$, are sufficient for obtaining connectivity constant $\omega$ that governs the leading exponential term in the scaling forms for $Z_{l}^{C}$ and $Z_{l}^{O}$, as well as constant $\lambda$ which appears in their correction terms. Whereas the leading term $\omega^{N_{l}}$ is the same for both closed and open HWs, correction term in both cases has the same stretched exponential form $\mu^{N_{l}^{\sigma}}$, but with different values for $\mu$ and $\mu_{O}>\mu_{C}$, indicating that number of open HWs is larger than the number of closed HWs. One should notice here that the fact that scaling form obtained for open HWs is determined by the behavior of the numbers $B_{l}$ and $E_{l}$ only, means that contribution of one- and two-leg HW conformations $\left(A_{-}^{-}, C^{-}, D\right.$ - and $H$-type walks), i.e. HWs with their ends in the interior of $G_{l}(b)$ fractal structures, is not significant. Furthermore, in sections 2 and 3 it was elaborated that the main mathematical reason for obtaining scaling forms with the stretched exponential correction term is the fact that $\lim _{l \rightarrow \infty} B_{l} / E_{l}=0$, meaning that entangled conformations (with large number of two-stranded parts) dominate over those in which HWs rarely return to already visited fractal generators.

Results of the study of HWs on 3d MSG fractals, presented in this paper, should be compared with the results recently obtained for other fractal lattices [7]. For $n$ simplex fractal lattices with even values of $n$ the same asymptotic behavior of the HW numbers was found, $\omega^{N} \mu^{N^{\sigma}}$, but with $\sigma=1 / d_{f}$, which differs from the formula derived here for $\sigma$. The existence of the term $\mu^{N^{\sigma}}$ was also related with the facts that: (1) entangled HWs prevail, and (2) HWs with "interior" terminating points do not contribute to the asymptotic behavior of HW numbers, as in the 3d MSG fractals case. For odd $n$, stretched exponential terms in the scaling forms for HWs on $n$-simplex fractals do not exist, HWs with "interior" terminating points do affect the scaling forms for the numbers of open HWs, and entangled conformations do not dominate in this case. For Given-Mandelbrot and 2d modified Sierpinski gasket fractals (which are two-dimensional generalizations of the Sierpinski gasket and 3-simplex fractal, respectively) stretched exponential terms are also absent, whereas again, one- and twoleg conformations are necessary for obtaining the scaling forms for open HWs, and, due to the specific topology of these lattices, only one-stranded HW conformations are possible. Therefore, results of the present study confirm the assumption that existence of the stretched exponential term is related with the issue of HW entanglement. However, the discrepancy between the formulas obtained for the exponent $\sigma$ for different fractal families, and, more generally, the question which properties of the underlying lattice and in what way determine $\sigma$, deserves further consideration. To this end we recall that stretched exponential terms were obtained for HWs on Manhattan [4], as well as for lowtemperature SAWs on square [8-11] and cubic [12] lattice. In this context, one should also mention the spiral SAWs on $2 \mathrm{~d}$ regular lattices [16] and lattice animals on some hierarchical lattices. Whereas, to the best of our knowledge, physical interpretation of the stretched exponential term for the $2 \mathrm{~d}$ spiral SAW models has never been proposed, its presence in the scaling forms obtained for lattice animals on hierarchical lattices was directly connected with the existence of the sets of sites with different coordination numbers [17]. Utilizing similar idea, in the case of low-temperature (collapsed) SAWs it 
was explicitly explained in [13] why term of the form $\mu^{N^{\sigma}}$, with $\sigma=(d-1) / d$ for regular lattices, should arise. However, as was elaborated in [6,7], direct application of such approach on HWs on fractal lattices does not give satisfactory result. Yet, the scaling forms obtained in this paper can be expound in the spirit of the physical reasoning given in [13], as will be explained in the following paragraph.

We shall first focus on closed HWs on $G_{l}(b)$ with the maximal number of twostranded $E$ conformations within the unit tetrahedrons. Such HWs represent maximally entangled closed (MEC) compact conformations and they accomplish the maximal possible connectedness between the generators $G_{l}(b)$ of all orders $l$. Now, from all the $N_{l}$ sites visited by such maximally entangled HW, observe those which belong to onestranded conformations ( $B$-steps) within the unit tetrahedrons, and which are directly connected only with the sites belonging to the same unit tetrahedron. For instance, vertices 9 and 10, or 15 and 16 in figure 2 represent examples of such sites. Since these sites are not directly connected with the other tetrahedrons of the lattice (via $\mathrm{HW}$ ), they are the maximally isolated sites within the maximally entangled closed HW conformation. It is shown in Appendix E that number $N_{l}^{C I}$ of such sites is equal to

$$
N_{l}^{C I}=\frac{1}{2} \frac{N_{G}-k_{C}-6}{N_{G}-3} N_{l}+2 \frac{N_{G}+k_{C}}{N_{G}-3} 4^{-\sigma} N_{l}^{\sigma},
$$

and one can easily see that, using this expression, scaling relation for the numbers od closed HWs can be transformed into the following form

$$
Z_{l}^{C} \sim \omega^{\prime N_{l}} \sqrt{\lambda}^{N_{l}^{C I}}, \quad \text { with } \omega^{\prime}=\omega / \lambda^{\frac{N_{G}-k_{C}-6}{4\left(N_{G}-3\right)}} .
$$

In a similar way (see Appendix E), one can show that scaling relation for open HWs can be expressed as

$$
Z_{l}^{O} \sim \omega^{N_{l}} \sqrt{\lambda}^{N_{l}^{O I}}
$$

where

$$
N_{l}^{O I}=\frac{1}{2} \frac{N_{G}-k_{C}-6}{N_{G}-3} N_{l}+2 \frac{k_{C}+3}{N_{G}-3} 4^{-\sigma} N_{l}^{\sigma}
$$

is the number of maximally isolated sites on $G_{l}(b)$ visited by maximally entangled open HW (which is an open HW with the maximal number of $E$-steps on unit tetrahedrons and with both loose ends being of type $C$, on all levels $l$ ). Comparing forms (4.2) and (4.3) with the scaling relations expected for HWs on homogeneous lattices, one can say that in this case terms $\sqrt{\lambda}^{N_{l}^{C I}}$ and $\sqrt{\lambda}^{N_{l}^{O I}}$ play the role of the stretched exponential correction term $\mu^{N^{\sigma}}$ in the case of homogeneous lattices. Indeed, for homogeneous lattices $N^{\sigma}$, with $\sigma=(d-1) / d$, is proportional to the number of sites on the lattice boundary, which have smaller number of neighbors than the bulk sites, and in that sense they are similar to the maximally isolated sites within the maximally entangled HWs on 3d MSG lattices. Therefore, one might generally expect that scaling relation for the number of HWs on nonhomogeneous lattices instead of stretched exponential correction term has a term $\mu^{N_{I}}$, where $N_{I}$ is the number of conveniently defined "maximally isolated sites". Relation between $N_{I}$ and the overall number of lattice sites $N$ depends on the 
particular topology of the lattice under study, which is the reason why different scaling forms, as functions of $N$, are obtained.

Conclusion of the previous paragraph can be supported by performing a similar analysis in the case of HWs on previously studied fractal families [7]. For instance, for $n$-simplex fractals maximally isolated sites can be defined in a similar way as for 3d MSG fractals, i.e. these are the sites which are directly connected by maximally entangled HW only with the sites within the same fractal generator they belong to. Here, maximally entangled HWs are those that contain maximal number of maximally-stranded HW parts. Then, it can be shown [18] that $N_{l}^{C I}=\frac{n}{2} N_{l}^{\sigma}$ and $N_{l}^{O I}=\left(\frac{n}{2}-1\right) N_{l}^{\sigma}$, where $\sigma=\ln 2 / \ln n$. Since overall numbers of closed and open HWs on $n$-simplex fractal with even $n$ asymptotically behave as $Z_{l}^{C} \sim \omega^{N_{l}} \lambda_{B}^{\frac{n}{2} N_{l}^{\sigma}}$ (see (5.8) in [7]) and $Z_{l}^{O} \sim \omega^{N_{l}} \lambda_{B}^{\frac{n-2}{2} N_{l}^{\sigma}}$ (formula (5.14) in [7]), respectively, they immediately fit into the scaling relation $\omega^{\prime N} \mu^{N_{I}}$, where $\omega^{\prime}=\omega$ and $\mu=\lambda_{B}$ for both types of HWs. For odd values of $n$, however, it can be shown [18] that number $N_{I}$ of maximally isolated sites, for both open and closed HWs, is proportional to $N_{l}$, so that any term of the form $\mu^{N_{I}}$ can only contribute to the leading term $\omega^{N}$. This certainly is in accord with the scaling forms found in [7] for odd $n$, which do not have stretched exponential corrections.

To conclude, we may say that presented exact study of Hamiltonian walks on modified three-dimensional Sierpinski gasket fractals supports the idea that entangled conformations are of the most physical importance for the behavior of compact polymers in inhomogeneous media. The introduced concept of "maximally isolated sites" proved to be useful in physical interpretation of different scaling forms obtained for Hamiltonian walks on all so far studied fractals. Whether such concept can be applied on homogeneous and other nonhomogeneous lattices will be the matter of our further investigations.

\section{Acknowledgments}

This paper has been done within the Project No OI 141020B, funded by the Serbian Ministry of Science and Environmental Protection.

\section{Appendix A. Maximal number of E-steps within HW configurations}

In this Appendix we prove that numbers $k_{C}, k_{B}$ and $k_{E}$ are given by formulae (2.4) and (2.7). The number $k_{C}$ is the maximum number of $E$-steps within the closed HW configuration (see (2.3)), whereas the numbers $k_{B}$ and $k_{E}$ are related to the maximum number of $E$-steps within the HW configurations of $B$ and $E$-type (see (2.5)), i.e. the conformations that traverse the generator of order $l+1$ once or two times, respectively. By ,E-step" here we imply any HW conformation consisting of two mutually avoiding strands, both traversing the generator of order $l$ (see figure 2). In a similar way, ,"B-step” is any HW conformation that consists of one strand traversing $G_{l}(b)$.

According to our definition of the modified Sierpinski gasket fractals, generators 


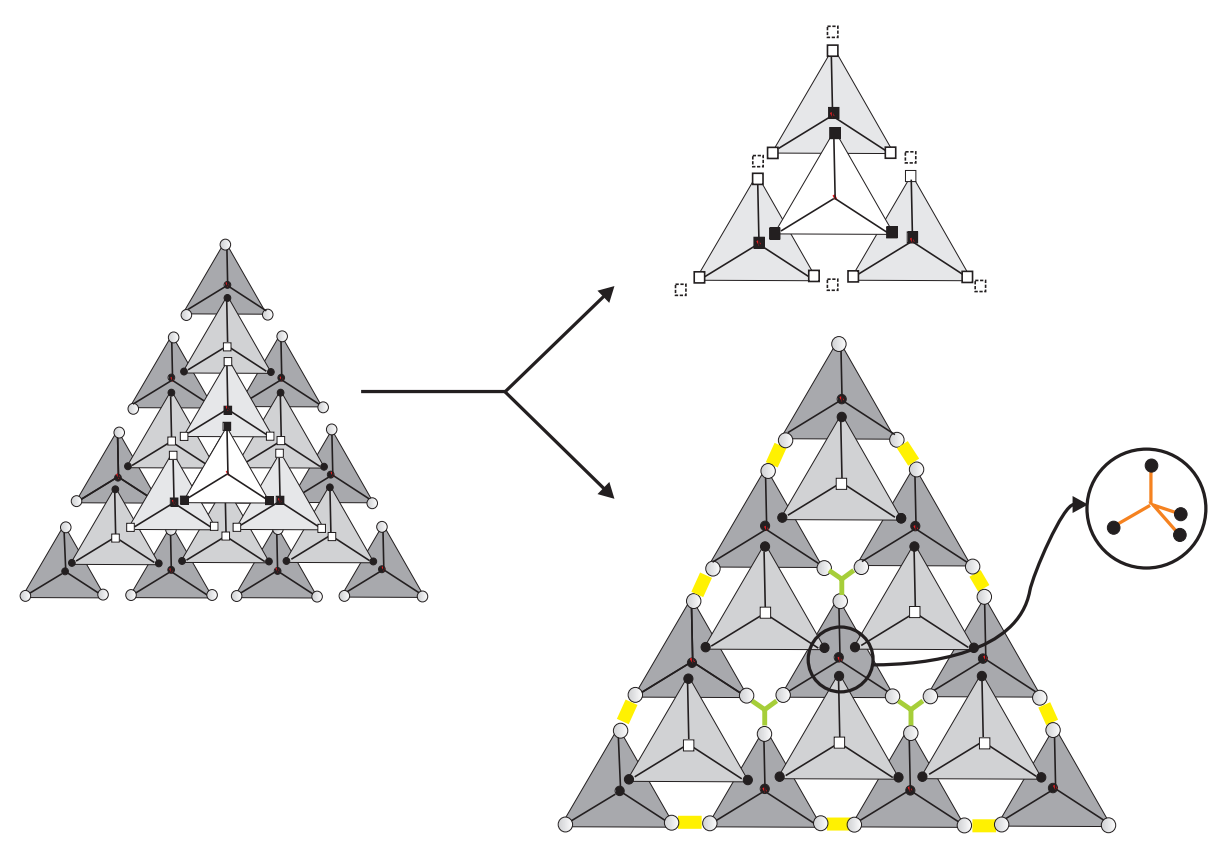

Figure A1. On the left-hand side of this picture generator $G_{l+1}(b)$ of order $l+1$, for $b=43 \mathrm{~d}$ MSG, is presented, as it is seen from above. Gray-shaded small tetrahedrons represent generators $G_{l}(4)$ of order $l$, lower layers being darker. Vertices lying in the same horizontal plane are indicated with the same symbol, being also the same for two adjacent planes containing vertices that belong to different generators. On the righthand side of the picture horizontal layers of tetrahedrons are split and slightly magnified in order that all vertices, as well as some junctions can be seen. In particular, twofold junctions in the lowest layer of vertices are indicated by yellow lines, whereas junctions joining three vertices are given in green color. There is only one four-fold junction - the corresponding four connected vertices are encircled, and in the magnified circle these vertices are presented as seen in three dimensions, with the junction indicated in orange color. One can check that overall number of junctions is $N_{J}=31$ (three twofold junctions for each of six $G_{l+1}(4)$ edges, three three-fold junctions for each of four $G_{l+1}(4)$ faces, and one four-fold interior junction), which certainly is in accord with formula (A.1).

$G_{l}(b)$ within the generator $G_{l+1}(b)$ are connected via infinitesimal junctions. Each of these junctions connects two, three or four neighboring $G_{l}(b)$ generators. ,Twofold" junction connects vertices of neighboring $G_{l}(b)$, both lying in the same edge of $G_{l+1}(b)$ (for instance, vertices 3 and 4 in figure 2 are joined by such junction). Vertices of neighboring $G_{l}(b)$ that lie inside the faces of $G_{l+1}(b)$ (such as sites 5,6 and 19 in figure 2) are connected by three-folded junction, whereas four-folded junction occur in the interior of $G_{l+1}(b)$, and can exist only for $b \geq 4$. In figure A1 we explicitly indicate some of the junctions connecting the vertices of the generators $G_{l}(4)$, within the generator $G_{l+1}(4)$. It is not difficult to show that the number of junctions which connect $N_{G}$ generators 
$G_{l}(b)$ into the generator $G_{l+1}(b)$ is equal to

$$
N_{J}=\frac{1}{6}(b+1)(b+2)(b+3)-4 .
$$

From the restraint that each site of the lattice has to be visited exactly once, follows that each junction (i.e. its middle point) can be traversed at most once. Now, suppose that closed HW conformation within $G_{l+1}(b)$ consists of $\alpha B$-steps and $\beta E$-steps. Each $G_{l}(b)$ is traversed by HW, which implies that

$$
\alpha+\beta=N_{G}=\frac{1}{6} b(b+1)(b+2) .
$$

Each $B$-step uses two junctions and each $E$-step uses four junctions. However, since each junction connects two $G_{l}(b)$ generators, the overall number of visited junctions is equal to $\alpha+2 \beta$. In addition, steps through corner $G_{l}(b)$ generators for the closed HW have to be of the $B$-type (see figure 2), which means that one of the three junctions for each of four corner generators is certainly not used. This implies that inequality

$$
\alpha+2 \beta \leq N_{J}-4=\frac{1}{6}(b+1)(b+2)(b+3)-8
$$

is satisfied. Then, from (A.2) and (A.3) directly follows that

$$
\beta \leq \frac{1}{2}(b+1)(b+2)-8
$$

i.e. the maximum number of $E$-steps within the closed $\mathrm{HW}$ conformation is indeed equal to

$$
\beta_{\max }=k_{C}=\frac{1}{2}(b+1)(b+2)-8 .
$$

Next, we consider a $B$-type HW configuration on $G_{l+1}(b)$ generator, with $\alpha B$ steps, and $\beta E$-steps. Steps through two corner $G_{l}(b)$ generators, at which HW starts or terminates, can be of either $B$ - or $E$-type. However, both remaining two corner $G_{l}(b)$ generators must be traversed by $B$-step (see figure A2), meaning that two of $N_{J}$ junctions are never used. On the other hand, each $B$-step uses two junctions, unless it is the first or the last step of the HW configuration, in which case it utilizes only one junction (since the corner vertex does not belong to any junction). Similarly, $E$-step which traverses interior $G_{l}(b)$ generators uses four junctions, whereas possible $E$-step through either the first or the last traversed $G_{l}(b)$ uses only three junctions. Suppose that both the first and the last visited corner generators contain $E$-step. Then, these two steps together use six junctions, and the remaining $(\beta-2)$, interior" $E$-steps use $4(\beta-2)$, whereas $\alpha B$-steps use $2 \alpha$ junctions. Since every junction can be traversed only once, and two consecutive steps share junctions, it follows that altogether $[6+4(\beta-2)+2 \alpha] / 2$ junctions are visited by such HW conformation. This implies the following inequality

$$
\frac{1}{2}[6+4(\beta-2)+2 \alpha] \leq N_{J}-2,
$$

which, together with the relation $\alpha+\beta=N_{G}$, gives

$$
\beta \leq N_{J}-N_{G}-1=\frac{1}{2}(b+1)(b+2)-5=\beta_{\max }=N_{G}-k_{B} .
$$




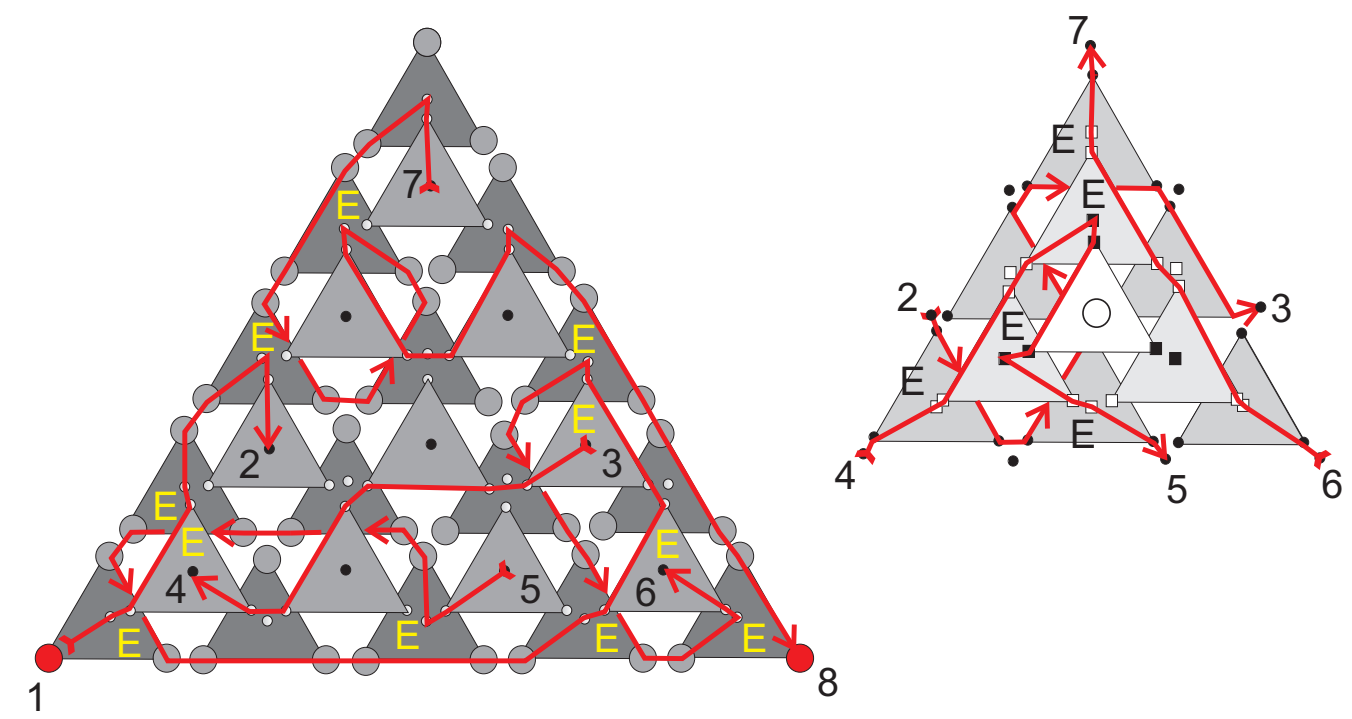

Figure A2. Generator $G_{l+1}(5)$, viewed from above, with the upper three layers of constitutive generators $G_{l}(5)$ (gray-shaded tetrahedrons-triangles) moved to the righthand side of the picture, for the sake of better visuality. Red oriented line represents a $B$-type HW configuration, which contains $16 E$-steps, i.e. the maximum possible number of them, given by formula (A.7). Remaining 19 steps are of the $B$-type, and they are depicted as straight lines connecting only two vertices of the traversed $G_{l}(5)$ generator, but it is implied that all sites within it are visited. The numbers in the figure denote points where HW leaves or enters the lower part of $G_{l+1}(5)$.

In the remaining two possible situations, when the first and the last $G_{l}(b)$ are traversed by either both $B$-steps, or one of them by $B$-step and the other by $E$-step, in a similar way it can be shown that inequality (A.7) holds as well.

As one can see in figure $\mathrm{A} 3$ an $E$-type HW conformation within $G_{l+1}(b)$ can use all three junctions of any corner $G_{l}(b)$, which happens when such $G_{l}(b)$ is traversed by $E$-step. This also means that all $N_{J}$ junctions within $G_{l+1}(b)$ can be utilized. Suppose that all four corner $G_{l}(b)$ generators are traversed by $E$-steps - altogether 12 junctions are used by these four $E$-steps. If the whole HW conformation contains $\beta E$-steps, the remaining $(\beta-4)$ interior $E$-steps take $4(\beta-4)$ junctions, whereas to all $\alpha B$-steps $2 \alpha$ junctions correspond. Every used junction connects two steps, therefore in this case inequality

$$
\frac{1}{2}[12+4(\beta-4)+2 \alpha] \leq N_{J},
$$

holds, which, again with $\alpha+\beta=N_{G}$, leads to

$$
\beta \leq N_{J}-N_{G}+2=\beta_{\max }=\frac{1}{2}(b+1)(b+2)-2=N_{G}-k_{E} .
$$

In a similar manner one can show that in the remaining four possibilities: one, two, three or four $B$-steps through the corner $G_{l}(b)$ generators, inequality (A.9) is valid again.

Note that formulae (A.5), (A.7) and (A.9) are not correct in the $b=2$ case. In this case all four constitutive $G_{l}(b=2)$ generators of the $G_{l+1}(b=2)$ generator are at its corners, and each of them is connected with each of the other three by two-folded 


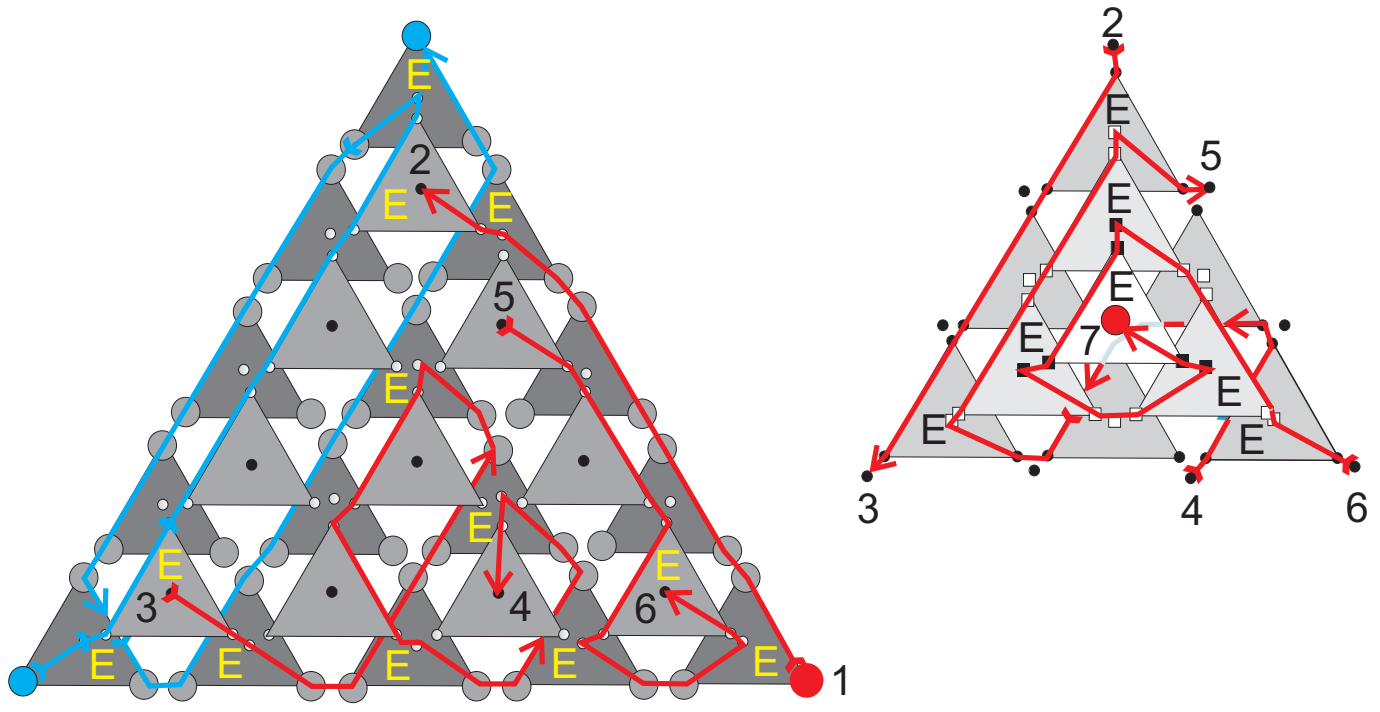

Figure A3. Example of $E$-type HW conformation on $G_{l+1}(5)$ with $19 E$-steps, which is the maximum possible number $\beta_{\max }$ of them, given by formula (A.9). The two strands of the conformation are depicted as oriented red and blue lines. The red strand starts at vertex labeled with number 1 , and terminates at vertex 7 , whereas the numbers 2 to 6 mark the sites at which the strand leaves or enters the second layer of $G_{l}(5)$ generators.

junction. Since two corner generators have a junction in common, the number of usable junctions in relations (A.3) and (A.6) is $N_{J}-2$ and $N_{J}-1$ instead of $N_{J}-4$ and $N_{J}-2$, respectively. If we represent each $G_{l}(2)$ by a point (vertex) then $G_{l+1}(2)$ represents a complete graph of four points. Since there can be neither subgraph with three points of degree three and one point of degree one, nor subgraph with two points of degree three and two of degree one, terms in relation (2.6) with $E^{3}$ and $E^{2}$ are not possible.

\section{Appendix B. Asymptotical behavior of the parameter $x_{l}=B_{l} / E_{l}$}

As it was stressed in section 2 asymptotical behavior of the parameter $x_{l}=B_{l} / E_{l}$ is of the greatest importance for obtaining the scaling form of HWs. Here we want to prove that assumption $p_{k_{E}+k}>m_{k_{E}+k+1}$, for $2 \leq k \leq k_{C}+5$, leads to the conclusion that $x_{l} \rightarrow 0$, when $l \rightarrow \infty$. Numbers $x_{l}$ satisfy the exact relation (2.8), from which follows that

$$
x_{l+1}-x_{l}=x_{l} \frac{-p_{k_{E}}-p_{k_{E}+1} x_{l}-p_{k_{E}+k_{C}+6} x_{l}^{k_{C}+6}-\sum_{k=2}^{k_{C}+5}\left(p_{k_{E}+k}-m_{k_{E}+k+1}\right) x_{l}^{k}}{\sum_{k=0}^{k_{C}+6} p_{k_{E}+k} x_{l}^{k}}<0,
$$

where we used the relation $k_{E}=k_{B}-3$, obtained in the previous Appendix, the fact that all coefficients $p_{k}$ and $m_{k}$ are positive, as well as the proposed inequality. This means that sequence of positive numbers $x_{l}$ is monotonically decreasing, and since $x_{0}=2$, it follows that this sequence has non-negative limiting value $c=\lim _{l \rightarrow \infty} x_{l}$. If $c>0$, again 
from (2.8), when $l \rightarrow \infty$, follows equation

$$
c=c^{3} \frac{\sum_{k=0}^{k_{C}+3} m_{k_{B}+k} c^{k}}{\sum_{k=0}^{k_{C}+6} p_{k_{E}+k} c^{k}},
$$

which, after dividing by $c$, can be transformed to

$$
0=p_{k_{E}}+p_{k_{E}+1} c+p_{k_{E}+k_{C}+6} c^{k_{C}+6}+\sum_{k=2}^{k_{C}+5}\left(p_{k_{E}+k}-m_{k_{E}+k+1}\right) c^{k} .
$$

However, the latter equation cannot be satisfied, since its right-hand side has the form of polynomial in $c$, with all its coefficients being positive numbers. Consequently, one concludes that $c=0$.

\section{Appendix C. Recursion relations for the numbers $A_{l}, C_{l}$ and $Z_{l}^{O}$}

In this Appendix we first show that polynomials $R_{i j}$, appearing in recursion relations (3.2) for numbers $A_{l}$ and $C_{l}$ of one-leg HW conformations, have the form (3.3), with $k_{i j}$ given by (3.8). It is obvious that dangling end-step of one-leg configuration within $G_{l+1}(b)$ can be either of $A$ - or $C$-type. The remaining part of the HW traverses the remaining $N_{G}-1$ generators $G_{l}(b)$, and consists of $\alpha B$-steps and $\beta E$-steps, so that equation

$$
\alpha+\beta=N_{G}-1
$$

is satisfied.

Consider an $A$-type HW configuration on generator $G_{l+1}(b)$. One of its end-steps is fixed at one of the four corner generators $G_{l}(b)$ and it can be either $B-$, $E-$, or $C$-step, respectively using one, three, or two junctions. Two of the remaining three corner generators are traversed by $B$-steps, each of them using two junctions of the corresponding $G_{l}(b)$, whereas its third junction cannot be traversed. The last corner generator is either traversed by a $B$-step, or it contains dangling $A$ - or $C$-step, and such steps use two, one, or three junctions, respectively. If a corner generator contains $A$-step, only one of its corresponding three junctions can be traversed. Now, suppose that ,corner" steps are $E B B B$, whereas the dangling $A$-step, using one junction, is in the interior generator. Such HW traverses $(3+2 \alpha+4(\beta-1)+1) / 2=\alpha+2 \beta$ junctions, and, since corner $B$-steps cannot use altogether three of all $N_{J}$ junctions, it follows that

$$
\alpha+2 \beta \leq N_{J}-3
$$

which with (C.1) gives

$$
\beta \leq N_{J}-N_{G}-2=\frac{1}{2}(b+1)(b+2)-6=k_{C}+2 .
$$

In a similar manner one can analyze all the other possibilities and obtain corresponding maximum value of $\beta$. From the table $\mathrm{C1}$, in which all of these possibilities, together 
Table C1. All possible situations for $A$-type HW configuration on $G_{l+1}(b)$, depending on the types of its dangling end-step, steps traversing corner generators $G_{l}(b)$ and endstep fixed in one of the corners. For each situation the corresponding numbers of passed and usable junctions, as well as the maximum possible value of number $\beta$ of $E$-steps, are given.

\begin{tabular}{lccccc}
\hline $\begin{array}{c}\text { dangling end-step } \\
\text { and its position }\end{array}$ & $\begin{array}{c}\text {,corner" } \\
\text { passing-steps }\end{array}$ & $\begin{array}{c}\text {,corner" } \\
\text { fixed-step }\end{array}$ & $\begin{array}{c}\text { number of } \\
\text { used junctions }\end{array}$ & $\begin{array}{c}\text { number of } \\
\text { usable junctions }\end{array}$ & $\beta_{\max }$ \\
\hline$A$ - interior $G_{l}(b)$ & $B B B$ & $E$ & $\alpha+2 \beta$ & $N_{J}-3$ & $N_{J}-N_{G}-2$ \\
$A$ - interior $G_{l}(b)$ & $B B B$ & $B$ & $\alpha+2 \beta$ & $N_{J}-5$ & $N_{J}-N_{G}-4$ \\
$A$ - corner $G_{l}(b)$ & $B B$ & $E$ & $\alpha+2 \beta$ & $N_{J}-4$ & $N_{J}-N_{G}-3$ \\
$A$ - corner $G_{l}(b)$ & $B B$ & $B$ & $\alpha+2 \beta$ & $N_{J}-6$ & $N_{J}-N_{G}-5$ \\
\hline$C$ - interior $G_{l}(b)$ & $B B B$ & $E$ & $1+\alpha+2 \beta$ & $N_{J}-3$ & $N_{J}-N_{G}-3$ \\
$C$ - interior $G_{l}(b)$ & $B B B$ & $B$ & $1+\alpha+2 \beta$ & $N_{J}-5$ & $N_{J}-N_{G}-5$ \\
$C$ - corner $G_{l}(b)$ & $B B$ & $E$ & $1+\alpha+2 \beta$ & $N_{J}-2$ & $N_{J}-N_{G}-2$ \\
$C$ - corner $G_{l}(b)$ & $B B$ & $B$ & $1+\alpha+2 \beta$ & $N_{J}-4$ & $N_{J}-N_{G}-4$ \\
$C$ - corner $G_{l}(b)$ & $B B B$ & $C$ & $1+\alpha+2 \beta$ & $N_{J}-4$ & $N_{J}-N_{G}-4$ \\
\hline
\end{tabular}

with the corresponding numbers of used and usable junctions, as well as $\beta_{\max }$ are given, one can see that $k_{11}=k_{12}=k_{C}+2$.

$C$-type HW configuration on $G_{l+1}(b)$ consists of two strands: one of $B$-type, i.e. with two ends fixed at two corners of the $G_{l+1}(b)$, and the other of $A$-type, with one end fixed at the third corner, whereas its second end is free, i.e. it can be anywhere within the $G_{l+1}(b)$, in some of interior generators $G_{l}(b)$, as well as in any of the four corner-generators $G_{l}(b)$. The three steps containing fixed ends can be of either $B$ - or $E$-type, whereas the corner-generator, which does not contain the fixed-end, is either traversed by a $B$-step or it contains the dangling $A$ - or $C$-step. The other relevant details of possible situations, regarding the type and the position of the step containing the dangling end, are listed in table C2. One can see that $\mathrm{HW}$ configurations with $A$ dangling end-step and maximal number of $E$-steps are those with the dangling end-step in the interior $G_{l}(b)$, whereas the three steps with ends fixed at corner-generators are of $E$-type, and the fourth corner-generator is traversed by a $B$-step, and thus conclude that $k_{21}=N_{J}-N_{G}+1=k_{C}+5$. Configurations with $C$ dangling end and maximal number of $E$-steps have also all three fixed-steps of $E$-type, while the dangling end is in the fourth corner-generator, therefore $k_{22}=k_{C}+5$.

In the remaining part of this Appendix we show that polynomials $F$ and $S$, appearing in the recursion relation (3.1) for the overall number $Z_{l+1}^{O}$ of open $\mathrm{HWs}$ on $G_{l+1}(b)$, have the form given by (3.5). Polynomials $F_{A A}, F_{A C}$ and $F_{C C}$ correspond to HWs whose two step-ends are two $A$-steps, one $A$ - end one $C$-step, and two $C$ steps, respectively. Since the whole HW configuration has $N_{G}$ steps, its ,interior" part has $N_{G}-2$ steps which are either of $B$ - or $E$-type, implying that $F_{X Y}$ are homogeneous polynomials in $B_{l}$ and $E_{l}$ of power $N_{G}-2$. In table C3 we list all possible arrangements of steps within open HW configuration with two one-leg end- 
Table C2. Description of possible $C$-type HW configurations on $G_{l+1}(b)$, depending on the types of dangling end-step, and the steps within corner-generators $G_{l}(b)$. For each situation the corresponding numbers of passed and usable junctions, as well as the maximum possible value of number $\beta$ of $E$-steps, are given.

\begin{tabular}{lccccc}
\hline $\begin{array}{c}\text { dangling end-step } \\
\text { and its position }\end{array}$ & $\begin{array}{c}\text {,corner" } \\
\text { fixed-steps }\end{array}$ & $\begin{array}{c}\text {,corner” } \\
\text { passing-step }\end{array}$ & $\begin{array}{c}\text { number of } \\
\text { used junctions }\end{array}$ & $\begin{array}{c}\text { number of } \\
\text { usable junctions }\end{array}$ & $\beta_{\text {max }}$ \\
\hline$A$ - interior $G_{l}(b)$ & $B B B$ & $B$ & $\alpha+2 \beta-1$ & $N_{J}-7$ & $N_{J}-N_{G}-5$ \\
$A$ - interior $G_{l}(b)$ & $B B E$ & $B$ & $\alpha+2 \beta-1$ & $N_{J}-5$ & $N_{J}-N_{G}-3$ \\
$A$ - interior $G_{l}(b)$ & $B E E$ & $B$ & $\alpha+2 \beta-1$ & $N_{J}-3$ & $N_{J}-N_{G}-1$ \\
$A$ - interior $G_{l}(b)$ & $E E E$ & $B$ & $\alpha+2 \beta-1$ & $N_{J}-1$ & $N_{J}-N_{G}+1$ \\
$A$ - corner $G_{l}(b)$ & $B B B$ & $A$ & $\alpha+2 \beta-1$ & $N_{J}-8$ & $N_{J}-N_{G}-6$ \\
$A$ - corner $G_{l}(b)$ & $B B E$ & $A$ & $\alpha+2 \beta-1$ & $N_{J}-6$ & $N_{J}-N_{G}-4$ \\
$A$ - corner $G_{l}(b)$ & $B E E$ & $A$ & $\alpha+2 \beta-1$ & $N_{J}-4$ & $N_{J}-N_{G}-2$ \\
$A$ - corner $G_{l}(b)$ & $E E E$ & $A$ & $\alpha+2 \beta-1$ & $N_{J}-2$ & $N_{J}-N_{G}$ \\
\hline$C$ - interior $G_{l}(b)$ & $B B B$ & $B$ & $\alpha+2 \beta$ & $N_{J}-7$ & $N_{J}-N_{G}-6$ \\
$C$ - interior $G_{l}(b)$ & $B B E$ & $B$ & $\alpha+2 \beta$ & $N_{J}-5$ & $N_{J}-N_{G}-4$ \\
$C$ - interior $G_{l}(b)$ & $B E E$ & $B$ & $\alpha+2 \beta$ & $N_{J}-3$ & $N_{J}-N_{G}-2$ \\
$C$ - interior $G_{l}(b)$ & $E E E$ & $B$ & $\alpha+2 \beta$ & $N_{J}-1$ & $N_{J}-N_{G}$ \\
$C$ - corner $G_{l}(b)$ & $B B B$ & $C$ & $\alpha+2 \beta$ & $N_{J}-6$ & $N_{J}-N_{G}-5$ \\
$C$ - corner $G_{l}(b)$ & $B B E$ & $C$ & $\alpha+2 \beta$ & $N_{J}-4$ & $N_{J}-N_{G}-3$ \\
$C$ - corner $G_{l}(b)$ & $B E E$ & $C$ & $\alpha+2 \beta$ & $N_{J}-2$ & $N_{J}-N_{G}-1$ \\
$C$ - corner $G_{l}(b)$ & $E E E$ & $C$ & $\alpha+2 \beta$ & $N_{J}$ & $N_{J}-N_{G}+1$ \\
$C$ - corner $G_{l}(b)$ & $B B C$ & $B$ & $\alpha+2 \beta$ & $N_{J}-6$ & $N_{J}-N_{G}-5$ \\
$C$ - corner $G_{l}(b)$ & $B E C$ & $B$ & $\alpha+2 \beta$ & $N_{J}-4$ & $N_{J}-N_{G}-3$ \\
$C$ - corner $G_{l}(b)$ & $E E C$ & $B$ & $\alpha+2 \beta$ & $N_{J}-2$ & $N_{J}-N_{G}-1$ \\
\hline
\end{tabular}

steps, together with the corresponding numbers of used and usable junctions. Using these data one can recognize arrangements with maximum possible number of $E$-steps, and conclude that this number is equal to $N_{J}-N_{G}-3=k_{C}+1$.

If an open HW has both its ends in the same $G_{l}(b)$, then this corresponds to $D_{-}$ or $H$-step within it, whereas remaining part of that HW consists of $\alpha B$-steps and $\beta$ $E$-steps such that $\alpha+\beta=N_{G}-1$. This means that $S_{D}$ and $S_{H}$ are homogeneous polynomials in $B_{l}$ and $E_{l}$ of power $N_{G}-1$. An $H$-step can exist only in interior $G_{l}(b)$, in which case all steps through corner-generators are of $B$-type, and four junctions can never be utilized. Since such HW takes $2+\alpha+2 \beta$ junctions, it follows that $\beta \leq N_{J}-N_{G}-5=k_{C}-1$. Finally, $D$-step can exist in any $G_{l}(b)$, either corner or interior one, but in both cases the number of used junctions is $1+\alpha+2 \beta$, and four junctions corresponding to corner-generators cannot be taken by such HW. Thus, one obtains that maximum number of $E$-steps is $\beta_{\max }=N_{J}-N_{G}-4=k_{C}$.

\section{Appendix D. Two-leg HW configurations}

Here we give some details of derivation of the conclusion that numbers of two-leg HW configurations are not needed for obtaining the scaling form of the overall number of 
Table C3. Possible steps arrangements of open HW configuration with one-leg ends, together with the corresponding numbers of used and usable junctions. The last column represents the maximum number of $E$-steps for each arrangement, obtained by the fact that number of used junctions cannot be larger than the number of usable junctions.

\begin{tabular}{ccccc}
\hline $\begin{array}{c}\text { steps through } \\
\text { corner-generators }\end{array}$ & $\begin{array}{c}\text { steps through } \\
\text { interior generators } \\
A A B B\end{array}$ & $\begin{array}{c}\text { number of used } \\
\text { junctions }\end{array}$ & $\begin{array}{c}\text { number of usable } \\
\text { junctions }\end{array}$ & $\beta_{\max }$ \\
$A B B B$ & $(\alpha-2) B, \beta E$ & $1+\alpha+2 \beta$ & $N_{J}-6$ & $N_{J}-N_{G}-5$ \\
$B B B B$ & $(\alpha-4) B, \beta E, 1 A$ & $1+\alpha+2 \beta$ & $N_{J}-5$ & $N_{J}-N_{G}-4$ \\
\hline$A C B B$ & $(\alpha-2) B, \beta E, 2 A$ & $1+\alpha+2 \beta$ & $N_{J}-4$ & $N_{J}-N_{G}-3$ \\
\hline$A B B B$ & $(\alpha-3) B, \beta E, 1 C$ & $2+\alpha+2 \beta$ & $N_{J}-4$ & $N_{J}-N_{G}-4$ \\
$C B B B$ & $(\alpha-3) B, \beta E, 1 A$ & $2+\alpha+2 \beta$ & $N_{J}-5$ & $N_{J}-N_{G}-5$ \\
$B B B B$ & $(\alpha-4) B, \beta E, 1 A, 1 C$ & $2+\alpha+2 \beta$ & $N_{J}-4$ & $N_{J}-N_{G}-3$ \\
\hline$C C B B$ & $(\alpha-2) B, \beta E$ & $3+\alpha+2 \beta$ & $N_{J}-2$ & $N_{J}-N_{G}-4$ \\
$C B B B$ & $(\alpha-3) B, \beta E, 1 C$ & $3+\alpha+2 \beta$ & $N_{J}-3$ & $N_{J}-N_{G}-3$ \\
$B B B B$ & $(\alpha-4) B, \beta E, 2 C$ & $3+\alpha+2 \beta$ & $N_{J}-4$ & $N_{J}-N_{G}-4$ \\
\hline
\end{tabular}

open HWs.

First we notice that recursion relation for the two-leg HW configuration of the type $D$, given in (3.4), follows from the fact that dangling ends of the two strands can reside either (1) in the same generator $G_{l}(b)$, thus forming $D$ - or $H$-step within it, or (2) in two different generators $G_{l}(b)$, thus forming two one-leg steps, either of $A$ - or $C$-type. It is not difficult to see that case (1) can be obtained by cutting a step of some $B$-type configuration. Therefore, each $B$-type configuration with $k B$-steps and $\left(N_{G}-k\right) E$-steps gives rise to $k D$-type configurations with $(k-1) B$-steps, $\left(N_{G}-k\right)$ $E$-steps and one $D$-step, which are obtained by cutting a $B$-step, whereas by cutting its $E$-steps (which can be done in two ways for each $E$-step) one can obtain $2\left(N_{G}-k\right)$ different $D$-type configurations with $k B$-steps, $\left(N_{G}-k-1\right) E$-steps and one $H$-step. In a similar way, by cutting an $E$-type HW configuration, one can obtain an $H$-type configuration. From these observations straightforwardly follows that

$$
\begin{array}{ll}
d_{D}(B, E)=\sum_{k=k_{B}}^{N_{G}} k m_{k} B^{k-1} E^{N_{G}-k}, & d_{H}(B, E)=2 \sum_{k=k_{B}}^{N_{G}}\left(N_{G}-k\right) m_{k} B^{k} E^{N_{G}-k-1}, \\
h_{D}(B, E)=\frac{1}{2} \sum_{k=k_{E}}^{N_{G}} k p_{k} B^{k-1} E^{N_{G}-k}, & h_{H}(B, E)=\sum_{k=k_{E}}^{N_{G}}\left(N_{G}-k\right) p_{k} B^{k} E^{N_{G}-k-1} .
\end{array}
$$

Applying a reasoning similar to one used in the previous Appendix, one can obtain that polynomials $d_{X Y}$ and $h_{X Y}$, corresponding to two-leg configurations with dangling ends in different generators, have the following forms

$$
d_{X Y}(B, E)=\sum_{k=0}^{k_{C}+4} s_{k}^{X Y} B^{N_{G}-2-k} E^{k}, \quad h_{X Y}(B, E)=\sum_{k=0}^{k_{X Y}} o_{k}^{X Y} B^{N_{G}-2-k} E^{k}
$$


with $s_{k}^{X Y}$ and $o_{k}^{X Y}$ being constant positive integers, $X Y=A A, A C, C C$, and

$$
k_{A A}=k_{C}+7=k_{A C}+1=k_{C C}+2 .
$$

Next, recursion relations (3.10) are obtained by dividing (3.4) with the recursion relation for $E_{l}$, given in (2.5). Therefore, coefficients $p_{i j}$ are defined as

$$
\begin{array}{ll}
p_{11}\left(x_{l}\right)=\frac{d_{D}\left(B_{l}, E_{l}\right) E_{l}}{E_{l+1}\left(B_{l}, E_{l}\right)}, & p_{12}\left(x_{l}\right)=\frac{d_{H}\left(B_{l}, E_{l}\right) E_{l}}{E_{l+1}\left(B_{l}, E_{l}\right)}, \\
p_{21}\left(x_{l}\right)=\frac{h_{D}\left(B_{l}, E_{l}\right) E_{l}}{E_{l+1}\left(B_{l}, E_{l}\right)}, & p_{22}\left(x_{l}\right)=\frac{h_{H}\left(B_{l}, E_{l}\right) E_{l}}{E_{l+1}\left(B_{l}, E_{l}\right)},
\end{array}
$$

and $t_{j i}$ as

$$
t_{1 i}\left(x_{l}\right)=\frac{d_{X Y}\left(B_{l}, E_{l}\right) E_{l}^{2}}{E_{l+1}\left(B_{l}, E_{l}\right)}, \quad t_{2 i}\left(x_{l}\right)=\frac{h_{X Y}\left(B_{l}, E_{l}\right) E_{l}^{2}}{E_{l+1}\left(B_{l}, E_{l}\right)},
$$

where $i=1,2,3$ corresponds to $X Y=A A, A C, C C$, respectively. Then, from (D.1) and (D.2) relations (3.11) directly follow.

For $l \gg 1, x_{l}$ tends to 0 , so that from (3.11) one obtains

$p_{11}\left(x_{l}\right) \approx k_{B} \frac{m_{k_{B}}}{p_{k_{E}}} x_{l}^{2}, \quad p_{12}\left(x_{l}\right) \approx 2\left(k_{C}+3\right) \frac{m_{k_{B}}}{p_{k_{E}}} x_{l}^{3}, \quad p_{21}\left(x_{l}\right) \approx \frac{k_{E}}{2} \frac{1}{x_{l}}$,

$p_{22}\left(x_{l}\right) \approx k_{C}+6, \quad t_{1 i}\left(x_{l}\right) \approx \frac{s_{k_{C}+4}^{X Y}}{p_{k_{E}}}, \quad t_{21}\left(x_{l}\right) \approx \frac{o_{k_{C}+7}^{A A}}{p_{k_{E}}} \frac{1}{x_{l}^{3}}$,

$t_{22}\left(x_{l}\right) \approx \frac{o_{k_{C}+6}^{A C}}{p_{k_{E}}} \frac{1}{x_{l}^{2}}, \quad t_{23}\left(x_{l}\right) \approx \frac{o_{k_{C}+5}^{C C}}{p_{k_{E}}} \frac{1}{x_{l}}$.

Finally, using these approximate forms while multiplying (3.10) with (2.9), relation (3.12) is obtained, with coefficients $a_{i j}$ equal to

$$
\begin{aligned}
& a_{11}=k_{B}\left(\frac{m_{k_{B}}}{p_{k_{E}}}\right)^{3}, \quad a_{12}=2\left(k_{C}+3\right)\left(\frac{m_{k_{B}}}{p_{k_{E}}}\right)^{3}, \\
& a_{21}=\frac{k_{E}}{2}\left(\frac{m_{k_{B}}}{p_{k_{E}}}\right)^{3}, \quad a_{22}=\left(k_{C}+6\right)\left(\frac{m_{k_{B}}}{p_{k_{E}}}\right)^{3},
\end{aligned}
$$

and

$$
b_{13}=\frac{s_{k_{C}+4}^{C C}}{p_{k_{E}}}\left(\frac{m_{k_{B}}}{p_{k_{E}}}\right)^{2} .
$$

\section{Appendix E. Maximally isolated sites within maximally entangled HWs}

In this Appendix we want to derive the formulae (4.1) and (4.4) for the numbers $N_{l}^{C I}$ and $N_{l}^{O I}$ of maximally isolated sites within the maximally entangled closed (MEC) and maximally entangled open (MEO) Hamiltonian walks, respectively. Since MEC HWs on the generator $G_{l}(b)$ of order $l$ are closed HWs with the maximal number of $E$ steps, and maximally isolated sites within MEC HW are "interior" vertices of $B$-steps made through unit tetrahedrons (i.e. not the vertices at which HW enters or exits the tetrahedron), number $N_{l}^{C I}$ is equal to

$$
N_{l}^{C I}=2 N_{l}^{B}
$$


where $N_{l}^{B}$ is the number of unit tetrahedrons traversed by $B$-steps. In order to calculate $N_{l}^{B}$, and consequently $N_{l}^{C I}$, we observe maximally entangled $B$-type (MEB) HWs, i.e. $B$-type HWs with the maximal number of $E$-steps, and, similarly, maximally entangled $E$-type (MEE) HWs. For MEB HW passing through $G_{l+1}(b)$ generator, we introduce label $N_{l+1}^{B B}$ for the numbers of unit tetrahedrons traversed by $B$-step. In a similar way, let $N_{l+1}^{E B}$ be the number of unit tetrahedrons of $G_{l+1}(b)$, traversed by $B$-step, for MEE HW. Then, from the recursion relation for the numbers $B_{l}$ and $E_{l}(2.5)$, follows the matrix recursion relation for the numbers $N_{l}^{B B}$ and $N_{l}^{E B}$ :

$$
\left(\begin{array}{c}
N_{l+1}^{B B} \\
N_{l+1}^{E B}
\end{array}\right)=\left(\begin{array}{cc}
k_{B} & N_{G}-k_{B} \\
k_{E} & N_{G}-k_{E}
\end{array}\right)\left(\begin{array}{c}
N_{l}^{B B} \\
N_{l}^{E B}
\end{array}\right) .
$$

Since $k_{B}$ and $k_{E}$ are given by (2.7), and for the unit tetrahedron one has $N_{0}^{B B}=1$ and $N_{0}^{E B}=0$, solving the obtained recursion relation one obtains

$$
\begin{aligned}
& N_{l}^{B B}=\frac{3+k_{C}}{N_{G}-3} 3^{l}+\frac{N_{G}-k_{C}-6}{N_{G}-3} N_{G}^{l}, \\
& N_{l}^{E B}=-\frac{N_{G}-k_{C}-6}{N_{G}-3} 3^{l}+\frac{N_{G}-k_{C}-6}{N_{G}-3} N_{G}^{l} .
\end{aligned}
$$

Taking into account that MEC HW on $G_{l+1}(b)$ consists of $k_{C}$ MEE HWs on $G_{l}(b)$ generators and $\left(N_{G}-k_{C}\right)$ MEB HWs on $G_{l}(b)$, as implied by (2.3) , it follows

$$
N_{l+1}^{B}=\left(N_{G}-k_{C}\right) N_{l}^{B B}+k_{C} N_{l}^{E B},
$$

and consequently

$$
N_{l}^{B}=\frac{N_{G}+k_{C}}{N_{G}-3} 3^{l}+\frac{N_{G}-k_{C}-6}{N_{G}-3} N_{G}^{l} .
$$

Since $N_{l}^{C I}=2 N_{l}^{B}, N_{l}=4 N_{G}^{l}$, and $\sigma=\frac{\ln 3}{\ln N_{G}}$, as given by (2.18), formula (4.1) straightforwardly follows. In a similar manner, from (3.1), (3.2), (3.3), (3.5), (3.8), and (E.4) for MEO one obtains (4.4).

\section{References}

[1] Vanderzande C, 1998 Lattice Models of Polymers (Cambridge: Cambridge University Press)

[2] Flory P J, 1956 Proc. R. Soc. A 23460

[3] Dill K A, 1999 Protein Sci. 81166

[4] Duplantier B and David F, 1988 J. Stat. Phys. 51327

[5] Bradley R M, 1989 J. Phys. A: Math. Gen. 22 L19

[6] Stajić J and Elezović-Hadžić S, 2005 J. Phys. A: Math. Gen. 385677

[7] Elezović-Hadžić S, Marčetić D and Maletić S, 2007 Phys. Rev. E 76011107

[8] Prellberg T, Owczarek A L, Brak R, and Guttmann A J, 1993 Phys. Rev. E 482386

[9] Owczarek A L, 1993 J. Phys. A: Math. Gen. 26 L647

[10] Bennet-Wood D, Brak R, Guttman A J, Owczarek A L, and Prellberg T, 1994 J. Phys. A: Math. Gen. 27 L1

[11] Baiesi M, Orlandini E and Stella A L, 2006 Phys. Rev. Lett. 96040602

[12] Grassberger P and Hegger R, 1995 J.Chem.Phys. 1026881

[13] Owczarek A L, Prellberg T and Brak R, 1993 Phys. Rev. Lett. 70951 
[14] Mayer J-M, Guez C and Dayantis J, 1990 Phys. Rev. B 42 660; Shakhnovich E and Gutin A, 1990 J.Chem.Phys. 93 5967; Pande V S, Joerg C, Grosberg A Yu and Tanaka T, 1994 J. Phys. A: Math. Gen. 27 6231; Kloczkowski A and Jernigan R L, 1997 Comput. Theor. Polym. Sci. 7 163; Kloczkowski A and Jernigan R L, 1998 J.Chem.Phys. 109 5134; Kloczkowski A and Jernigan R L, 1998 J.Chem.Phys. 109 5147; Jensen I, 2001 Comput. Phys. Commun. 142 109; Bousquet-Mélou M, Guttman A J and Jensen I, 2005 J. Phys. A: Math. Gen. 38 9159; Peto M, Sen T Z, Jernigan R L and Kloczkowski A, 2007 J.Chem.Phys. 127 044101; Jacobsen J L, 2007 J. Phys. A: Math. Gen. 4014667

[15] Ramakrishnan R, Pekny J F and Caruthers J M, 1995 J.Chem.Phys. 103 7592; Jaeckel A, Sturm J and Dayantis J, 1997 J. Phys. A: Math. Gen. 30 2345; Lua R, Borovinskiy A L and Grosberg A Yu, 2004 Polymer 45 717; Mansfield M L, 2006 J.Chem.Phys. 125 154103; Oberdorf R, Ferguson A, Jacobsen J L and Kondev J, 2006 Phys. Rev. E 74 051801; Jacobsen J L, 2008 Phys. Rev. Lett. 100118102

[16] Blöte H W J and Hilhorst H J, 1984 J. Phys. A: Math. Gen. 17 L111; Guttman A J and Wormald N C, 1984 J. Phys. A: Math. Gen. 17 L271; Guttman A J and Wallace K J, 1986 J. Phys. A: Math. Gen. 191645

[17] Knežević D, Djordjević K and Knežević M, 2007 J. Stat. Mech. P12007

[18] Lekić D, 2009 Private communication 